\title{
Universality in the Temperature Dependence of the Heat Capacity of Magnetic Solids
}

\author{
U. Köbler \\ Research Center Jülich, Institute PGI, 52425 Jülich, Germany \\ E-mail: u.koebler@fz-juelich.de
}

Received 22 June 2020, Accepted 14 August 2020

\begin{abstract}
It is shown that the temperature dependence of the heat capacity of the magnetic solids can completely be described by a sequence of universal power functions of temperature. Characteristic for universality is that each power function holds over a finite temperature range and has a rational exponent. The analytical change from one to the adjacent power function is a typical crossover event. Universality reveals that the temperature dependence of the heat capacity is determined by a boson field whereas the absolute values are given by all magnetic and non-magnetic inter-atomic interactions. Universality for temperatures outside the critical range at $\mathrm{T}_{\mathrm{c}}$, i.e. for temperatures for which the phonons dominate the heat capacity has to be characterized as non-intrinsic, arising from interactions of the phonons with the bosons of the continuous magnetic medium. As we have shown earlier, the bosons of the continuous magnetic solid are essentially magnetic dipole radiation generated via stimulated emission by the precessing spins. We have called them Goldstone bosons. The interactions of the Goldstone bosons with the magnons modify the wavevector dependence of the magnons. For cubic crystals the dispersions along $[\zeta, 0,0]$ direction are essentially as for the linear spin chain, i.e. one-dimensional. As the different rational exponent values in the temperature power function of the heat capacity show, there exists a number of distinct modes of interaction between the Goldstone boson field and the phonons. The actual exponent depends additionally on the proportion between the magnetic and the non-magnetic energy contributions and therefore changes with temperature. The observed exponents are, however, difficult to interpret.
\end{abstract}

Keywords: Ordered boson fields; magnetic domain patterns; critical dimensionality.

\section{Introduction}

As is well-known, the magnetic ordering transition gives rise to a peak in the heat capacity. Since development of the Renormalization Group (RG) theory [1] we know that the critical magnetic dynamics is the dynamics of a boson field. At the critical temperature the boson field orders. As a consequence, the peak in the heat capacity has to be attributed to the boson field. Ordering of the spin system is a consequence of the ordering of the boson field.

The bosons are the excitations of the continuous magnetic solid. We have called them Goldstone bosons [2]. Experimental evidenced could be obtained that the Goldstone bosons are essentially magnetic dipole radiation, generated by the rotating transverse components of the precessing spins [3]. From the identical critical behavior of ferromagnets and of antiferromagnets it immediately follows that the exchange interactions between the spins are of no importance for the critical magnetic dynamics [4]. This means, all thermal energy is in the boson field and not in the system of the interacting spins. As a consequence, the magnetic ordering transition is not driven by the exchange interactions between the spins but by the boson field. According to the symmetry selection principle of relevance of the RG-theory, either the bosons with continuous translational symmetry or the magnons with discrete and periodic translational symmetry can host the thermal energy and thus determine the dynamics.

Condition for a definite symmetry classification is that the thermal energy can change between the two systems. This requires a finite interaction 
between them. The transfer of the thermal energy from the system of the interacting spins to the boson field occurs at the crossover from the CurieWeiss susceptibility to the critical susceptibility [5].

Universality of the critical behavior results from the fact that the bosons propagate ballistic, that is, independent of all microscopic details such as the spin structure and largely also the lattice structure. In fact, universality is the thermodynamic behavior of a boson field. Since integer and halfinteger spins precess somewhat different, the generated boson fields are correspondingly different [6,7]. As a consequence, the critical exponents of the boson-driven ordering transition are different for magnets with an integer and with a half-integer spin [7] and differ distinctly from the predictions of the atomistic models [8]. For instance, mean field critical behavior is specific to the magnets with a half-integer spin and a threedimensional boson field [5-7]. A realistic field theory of the critical magnetic behavior, conforming to these experimental findings is, however, missing [9].

Typical for the ordered boson field are domains [7]. The ordered bosons are in coherent states, and propagate no longer isotropic in space but along the few domain axes only. In this way the spatially isotropic symmetry of the disordered boson field gets broken upon crossing the magnetic ordering transition. Essential for the broken symmetry and for the perfect one-dimensional boson field within each magnetic domain is that the bosons get generated by stimulated emission $[3,7]$. The onedimensional boson field within each domain resembles the radiation field of a LASER. The magnetic ordering transition can be compared with the threshold for the onset of stimulated emission of a LASER.

Due to a finite interaction between the ordered boson field and the spins, the spin system gets ordered. The one-dimensional boson field within each domain aligns all spins rigidly collinear to its axis, in spite of ubiquitous local exchange anisotropies that are, as all microscopic interactions, not relevant for the dynamics. Only the local spin order, either parallel or antiparallel, is determined by the exchange interactions between the spins, but this microscopic detail has no effect on the boson field. In other words, the functionality of bosons and magnons is clearly distinguished. Moreover, the coherence of the boson field stabilizes a perfect long-range magnetic order over a distance given by the coherence length of the bosons that corresponds to the linear dimension of the domains. Note that magnons are the local excitations of the discrete atomic lattice, and provide no evidence of a coherent long-range order [7]. Since magnons occur above the magnetic ordering temperature as well [7], they are indicative of a short-range order only.

In the magnets with a pure spin moment, such as in tetragonal $\mathrm{MnF}_{2}$, the collinear and coherent spin order is much stronger stabilized than it can be expected if the nearly isotropic exchange interactions [10] would be the relevant excitations. For magnets with a pure spin moment, i.e. with a $\mathrm{g}$-factor of $\mathrm{g} \approx 2$, the spin-flop field [11] and the magnon gap [10] provide a measure of the bosonstabilized collinear spin order [6]. For $\mathrm{T} \rightarrow \mathrm{T}_{\mathrm{c}}$ the spin order gets unstable and the gap tends to zero. For $\mathrm{MnF}_{2}$ with an axial lattice structure, and only one-domain type along symmetry axis, the stabilized one-dimensional spin structure lets the longitudinal susceptibility (along the tetragonal axis) tend to zero for $\mathrm{T} \rightarrow 0[12,13]$. Note that in the framework of the atomistic models, $\chi \rightarrow 0$ for $\mathrm{T} \rightarrow 0$ can be explained by a very strong axial singleparticle anisotropy only, which is absent in the magnets with pure spin magnetism [6,13]. It is the one-dimensional boson field of the single domain material $\mathrm{MnF}_{2}$ that provides the ordered spin system with a large axial anisotropy, thus giving rise to a surprisingly large magnon gap of 1.06 $\mathrm{meV}$ [10] and a spin-flop field of $120 \mathrm{kOe}$ [11]. $\mathrm{MnF}_{2}$ therefore provides the opportunity to study the properties of the isolated magnetic domain.

The boson-magnon interaction does not only generate a magnon gap, it modifies the wavevector dependence of the magnon excitation spectra for all q-values $[6,7,14]$. Since magnon propagation is restricted to the volume of the individual domain, within which the spin alignment is perfectly one-dimensional, the magnon dispersion is essentially as for the linear spin chain $[7,14]$. Only the absolute magnon energies are given by the microscopic interactions between the spins. Since the wave-vector dependence of the magnons is determined by the Goldstone bosons, it can be understood that the spin dynamics is also determined by the Goldstone bosons, and that the spontaneous magnetization follows universal power functions of temperature with rational exponents that are defined by the Goldstone boson field [6,7].

However, the linear chain dispersion of the magnons pertains to the individual domain and is 
not typical for the dimensionality of the global boson field. A two-dimensional or threedimensional global boson field results by some dynamic coupling of the one-dimensional boson fields of the differently oriented magnetic domains [15]. The dimensionality of the global boson field can be recognized from the number of inequivalent domain orientations, provided there is a finite dynamic coupling between the domains, which is, however, not always given [15]. The inter-domain coupling is different for magnets with an integer and with a half-integer spin, and determines the dimensionality of the global boson field and, therefore, the observed critical exponents [6,7]. The one-dimensional boson field within each domain remains essentially unchanged, and is responsible for the collinear spin order but not for the dynamics. Note that this definition of the dimensionality of ordered magnets refers to the domain configuration, and to the resulting dynamics of the global boson field and is completely different from the classification of the atomistic models that do not consider magnetic domains, and distinguish between the dimensionality of the spin and between an idealized, mostly not perfect realized dimensionality of the exchange interactions $[8,16]$. The only condition for boson dynamics is that the dimensionality of the spin must be three, in order that the spins are able to precess and can generate field quanta. Ising spins, for instance, do not precess and therefore are unable to generate Goldstone bosons. As a consequence, in Ising magnets the boson field gets not populated with field quanta, and the dynamics is determined by the atomistic exchange interactions instead by a boson field [3]. However, true Ising magnets are extremely rare. Only for the two known, real 2dIsing magnets, $\mathrm{K}_{2} \mathrm{CoF}_{4}$ and $\mathrm{Rb}_{2} \mathrm{CoF}_{4}$, it could be evidenced experimentally that the spontaneous (sublattice)magnetization is perfectly described by Onsager's exact solution of the 2d-Ising Hamiltonian [17-19]. Note that the conventional classification by the term $2 \mathrm{~d}$-Ising model means two-dimensional exchange interactions and a onedimensional spin.

Characteristic for the magnetic order due to exchange interactions, is the absence of domains [19]. Connected with this fact is that the collinear spin order is not well stabilized. The magnetic ordering transition is a less sharp event [20]. For the two 2d-Ising magnets $\mathrm{K}_{2} \mathrm{CoF}_{4}, \mathrm{Rb}_{2} \mathrm{CoF}_{4}$, the susceptibility along the axis of the ordered Ising spins is finite for $\mathrm{T} \rightarrow 0$ [20]. For all other magnets with precessing three-dimensional spins, the generated boson field is the relevant excitation system and defines the universal dynamics. As a consequence, the Heisenberg model is not realized in nature [8]. The presence of domains provides another clear evidence for boson dynamics.

Boson dynamics reveals furthermore from the finite width of the critical range and from the rational values of the critical exponents $\alpha$ (heat capacity), $\beta$ (spontaneous magnetization), $\gamma$ (susceptibility), $v$ (inverse correlation length), etc. [7]. Note that the critical power functions calculated by the atomistic models on the basis of Hamiltonians, hold asymptotically at the critical temperature only, and have exponents that are not necessarily rational numbers $[8,17]$. Moreover, the actual, the boson driven ordering transition is at a lower temperature compared to the hypothetical exchange driven ordering transition as it would be determined by the absolute magnitude of the magnon energies [5]. For the ferromagnets, the ordering transition gets decreased from the CurieWeiss temperature $\Theta$ to the actual ordering temperature, $\mathrm{T}_{\mathrm{C}}$, upon crossover from atomistic dynamics to boson dynamics that occurs at the crossover from Curie-Weiss susceptibility to critical susceptibility [5].

It is, however, not possible to conclude from the critical exponent $\alpha$, observed in the heat capacity at $T_{c}$, on the dimensionality of the global boson field. This is essentially because the heat capacity is an integral quantity and includes magnetic and non-magnetic contributions that are not independent of each other but interact significantly. Only the critical exponents of the spontaneous (sublattice)magnetization and of the (staggered) susceptibility are intrinsically magnetic and are characteristic for the dimensionality of the global boson field [15]. As will be shown in this communication, the interaction of the Goldstone bosons with the nonmagnetic degrees of freedom of the phonons affects the critical behavior of the heat capacity and, as a consequence, modifies the critical exponent $\alpha$. The effective interaction between the magnetic and the elastic degrees of freedom depends not only on the coupling strength between bosons and phonons but additionally on how strong the phonons are thermally exited. An approximate measure of how strong the phonons are excited at the critical temperature $T_{c}$ is given by the expression $T_{\mathrm{c}} / \Theta_{\mathrm{D}}$ with $\Theta_{\mathrm{D}}$ as Debye temperature. This quantity is certainly not quantized and cannot be the reason for the 
observed discrete and rational values of $\alpha$. Additionally, the coupling strength between bosons and phonons will be different for different materials. The observed rational critical exponents $\alpha$ therefore vary over a wide range. However, the general observation of universality illustrates the dominant role of the bosons for the dynamics of the coupled system of the elastic and magnetic degrees of freedom.

Due to the finite boson-phonon interaction is a quantitative separation into magnetic and nonmagnetic heat capacity contributions at $T_{c}$ not possible, and, fortunately, not necessary because the Goldstone bosons govern the temperature dependence of the total heat capacity. In other words, in contrast to the common practice in the earlier analyses of the critical heat capacity data, the magnetic and the non-magnetic heat capacity contributions do not superimpose linearly [21-24]. The observed heat capacity is not given by a sum of different functions of temperature but by a sequence of sectionally different universal power functions of temperature. Only for non-interacting systems, the heat capacities superimpose. This, however, is observed in the metallic solids only. As is well-known, the heat capacity of the Debye bosons $\left(\sim \mathrm{T}^{3}\right)$ and the heat capacity of the bosons of the continuous metallic solid $(\sim \mathrm{T})$ superimpose at very low temperatures [25]. Since the bosons of the continuous metallic solid do nearly not interact with the elastic degrees of freedom, they have a large mean free path [26]. This is different for the Debye bosons that interact with the phonons [26,27].

The conceptual shortcomings of the conventional data analyses of the critical heat capacity had severe consequences on the fitted values for the critical exponent $\alpha$ and on their interpretation. In particular, data analysis was made under the premise that the heat capacity is finite at $\mathrm{T}_{\mathrm{c}}$, and that the critical exponents $\alpha$ are identical above and below $\mathrm{T}_{\mathrm{c}}$ [8], in alleged agreement with the atomistic models. The here presented analyses of published heat capacity data demonstrate the inadequacy of the atomistic models, and result in dramatically different values for the critical exponent $\alpha$.

Interactions of the magnetic system with the elastic degrees of freedom are well-known from the phenomenon of magnetostriction $[6,28,29]$. In the thermal lattice parameter variation due to spontaneous magnetostriction, universal power functions of absolute temperature with rational exponents can also be identified [6]. Commonly, the spontaneous magnetostriction is weak above the magnetic ordering temperature but strong below the magnetic ordering temperature, i.e. not symmetric with respect to the ordering temperature [6]. This corresponds to the different critical exponents $\alpha$ above and below $\mathrm{T}_{\mathrm{c}}$. As the discrete values of the critical exponent $\alpha$ show, there are only well-distinguished modes of interaction between the Goldstone bosons and the phonons. The boson-phonon interaction therefore can be considered as quantized. It is evident that the assumed contribution of the phonons to the total heat capacity is a continuous and steadily increasing function of temperature. Nevertheless, the boson-controlled total heat capacity reacts in discrete steps on the continuous variations of the non-magnetic (and non-relevant) background. As we know from RG-theory, when non-relevant energy degrees of freedom have increased beyond some threshold, they can induce an analytical crossover event in the temperature dependence of the relevant system. This crossover can be either a sudden change in the pre-factor of the universal power function (amplitude crossover) [5] or in both, the pre-factor and the exponent [6]. Each crossover is additionally associated with a change of the absolute constant. Note that only the asymptotic "critical" power function for $\mathrm{T} \rightarrow 0$ includes no absolute constant.

As a consequence of the increasing importance of the phonons as a function of an increasing temperature, consecutive crossover events can be induced in the temperature dependence of the total heat capacity. The observed universality in temperature regions with a phonon dominated heat capacity has to be characterized as non-intrinsic, arising from the interactions of the phonons with the Goldstone bosons. The fact that universality is observed far above $T_{c}$ proves that the Goldstone bosons can dominate the temperature dependence of the heat capacity also when they have no heat capacity. No heat capacity means that the dispersion relation is thermally not populated. This is as for the disordered Debye boson field in the non-magnetic solids [27]. The dispersion relation of the Debye bosons is populated thermally only up to $\sim 10 \ldots 30 \mathrm{~K}$. Nevertheless, the Debye bosons provide the heat capacity of the phonons with universal power functions of temperature up to the Dulong-Petit (D-P) limit [27].

In the critical range near $T_{c}$, the universal power functions in the heat capacity are functions of $\mid \mathrm{T}$ $\mathrm{T}_{\mathrm{c}}$, outside the critical range they are functions of absolute temperature. The universal power 
functions of absolute temperature show that $\mathrm{T}=0$ is a critical temperature, completely equivalent to a finite ordering temperature. Moreover, since outside the critical range at $\mathrm{T}_{\mathrm{c}}$ only power functions of absolute temperature occur, $\mathrm{T}=0$ is the only other critical temperature in addition to the finite magnetic ordering temperature. The universal power functions of absolute temperature have to be ascribed to the boson field with the critical temperature of $\mathrm{T}=0$. This boson field defines the temperature dependence of the heat capacity for all temperatures, except for the rather narrow critical range. On the high- and lowtemperature side of $T_{c}$ the critical range is limited by the analytical crossover events from the power functions of the argument $\left|\mathrm{T}-\mathrm{T}_{\mathrm{c}}\right|$ to the power functions of absolute temperature. In other words, the boson field with the critical temperature $T=T_{c}$ is the relevant excitation system over a relatively narrow temperature range only.

Note that boson fields with a critical temperature of $\mathrm{T}_{\mathrm{c}}=0$ are not ordered. This applies to the Debye boson field (sound waves). Nevertheless, the heat capacity of the Debye boson field shows universality for $\mathrm{T} \rightarrow 0$ and is given for all solids by the famous $\mathrm{T}^{3}$ function that holds over a finite temperature range, commonly up to $\mathrm{T}=10 \ldots 30 \mathrm{~K}$. At this temperature, thermal energy gets transferred to the phonon system [30]. This crossover marks the width of the critical range at $\mathrm{T}=0$. Essential for the occurrence of the same $\mathrm{T}^{3}$ function in solids with different lattice structures is that there are no domains in the disordered Debye boson field. In this respect the disordered Debye boson field can be considered as isotropic. Note, that the dimensionality of the ordered Goldstone boson field and therefore the universal exponents, depend on the domain configuration which is coupled to the lattice structure. In axial crystals, commonly only one domain type along the symmetry axis occurs. The global Goldstone boson field then is that of the isolated domain, i.e. one-dimensional. In cubic crystals, the global Goldstone boson field is three-dimensional, provided the magnetic domains along $\mathrm{x}-$, $\mathrm{y}$ - and $\mathrm{z}$ axis are coupled dynamically [15]. Since the domain configuration of the non-cubic materials is not very stable and can depend on the strain in the sample, the universality classes, and therefore the critical exponents, can be meta-stable [6]. Metastability of the domain configuration is wellknown from the ferromagnets that can be transformed into the mono-domain state by the application of the moderate demagnetization field
[32]. In a similar way can the mosaic structure of the non-magnetic, axial crystals easily be manipulated by the application of moderate pressure [31].

It belongs to the important issues of RG theory that in the vicinity of a critical temperature, either $\mathrm{T}=0$ or a finite ordering temperature, the dynamics is exclusively due to bosons, i.e. universal. In the temperature range of the "critical" $\mathrm{T}^{3}$ function of the Debye boson field, the atomistic phonons are not relevant and do not contribute at all to the heat capacity $[14,27]$. The dispersion of the phonons is thermally not populated. This is as for the exchange interactions between the spins, that are completely excluded from the critical paramagnetic dynamics above the magnetic ordering temperature (and for all lower temperatures as well) [5-7]. Exclusion of the exchange interactions is the basis of the universality of the critical magnetic behavior. Only for temperatures beyond the crossover from the critical paramagnetic susceptibility to the Curie-Weiss susceptibility, the atomistic exchange interactions are the relevant excitations [5].

Non-relevance of the Debye bosons for temperatures of higher than $\sim 20 \mathrm{~K}$ means that the dispersion relation of the Debye bosons is no longer thermally populated $[26,27,30]$. The thermal energy now is in the system of the vibrating atoms (phonons). The absolute values of the heat capacity then are given by the total number of the atomic degrees of freedom and by the inter-atomic interaction strengths. Nevertheless, as we have shown recently, the temperature dependence of the heat capacity of the non-magnetic solids is determined for all temperatures by the Debye bosons that furnish the heat capacity of the lattice(!) with non-intrinsic universal power functions of absolute temperature up to the Dulong-Petit (D-P) limit [27].

A boson-controlled temperature dependence of the heat capacity of the phonons requires a finite interaction between bosons and phonons. This interaction modifies the dispersion relations of both systems: the dispersion of the Debye bosons becomes a weaker than linear function of wavevector and the wave-vector dependence of the phonon dispersions gets composed of two analytically different sections $[14,27,30]$. For low q-values the dispersion of the acoustic phonons agrees over a finite q-range with the linear dispersion of the Debye bosons [27,30]. Identical dispersions of Debye bosons and phonons means that the two dispersion relations have attracted 
each other. This is usually the case when there is an interaction between excitations with different (translational) symmetries. Crossing then is forbidden. For larger q-values an analytical crossover to a sine function of wave vector occurs in the dispersion of the acoustic phonons along the $\left[\begin{array}{lll}1 & 0 & 0\end{array}\right]$ direction $[27,30]$. Since the sine function is the dispersion of the linear atomic chain it is suggestive to assume that the generation of the Debye bosons is, as the generation of the Goldstone bosons, by stimulated emission. However, nothing is known about the spontaneous generation (or absorption) of Debye bosons by individual atoms.

As a consequence, in contrast to the atomistic models [33], the phonon dispersion relations cannot be understood neglecting the interaction with the Debye bosons. This interaction is active for all thermal energies, also for those for which the dispersion relation of the Debye bosons is thermally not populated, i.e., for temperatures of $\mathrm{T}>10 \ldots 30 \mathrm{~K}$. For these temperatures, the Debye bosons are physically not present and can be considered as virtual states. Nevertheless, Debye bosons (sound waves) can be excited out of thermal equilibrium for all temperatures, up to melting point. Since the Debye bosons modify the wave-vector dependence of the phonons it appears plausible that they determine the temperature dependence of the heat capacity of the phonons as well $[14,27]$. Quite generally, bosons seem to be the dominant (or relevant) excitations to determine the temperature dependence of the thermodynamic observables, also for temperatures for which the atomic degrees of freedom dominate the absolute values of the heat capacity. This will be further confirmed by the magnetic solids investigated here.

The interplay of the atomistic and of the bosonic degrees of freedom is well-known for the magnetic solids: the absolute values of the spontaneous magnetization are given by the atomic magnetic moments but the dynamics, for instance, the temperature dependence of the spontaneous magnetization is determined by the Goldstone boson field and exhibits universal power functions of temperature [5-7]. In particular, the thermal decrease of the spontaneous magnetization with respect to $\mathrm{T}=0$ is given by the heat capacity of the Goldstone boson field at $\mathrm{T}=0$. A spin dynamics that is controlled by the Goldstone bosons requires a finite boson-magnon interaction. This interaction has a similar effect as the Debye boson-phonon interaction in the non- magnetic solids, in that the dispersion of the magnons assumes essentially the dispersion of the linear spin chain $[7,14]$. Note that according to spin wave theory, the linear spin chain is not ordered at any finite temperature [34]. This proves again the inapplicability of the spin-wave theory that restricts on the exchange interactions and neglects the energy degrees of freedom of the continuous magnetic solid. As for the acoustic phonons [14,27], the dispersion of the magnons cannot be understood on the basis of the atomistic interactions [7]. On the other hand, non-relevance of the atomistic interactions is condition for universality.

Since the heat capacity is an integral quantity, all energy degrees of freedom contribute potentially to the absolute value of the heat capacity. In the magnetic solids, this are the Debye bosons, the Goldstone bosons, the phonons and the magnons. In the metallic magnets, the heat capacity of the bosons of the continuous metallic solid has to be considered additionally $[25,26]$. The decisive difference to the conventional, exclusively atomistic point of view is, that one has to distinguish between the different translational symmetries of the different sub-systems and the effects due to their interactions. As a consequence of these interactions, thermal energy can change between the different sub-systems, and can concentrate in one sub-system. The total heat capacity therefore is not given by a linear superposition of all individual heat capacity contributions, assuming that all dispersion relations are thermally populated according to the Boltzmann factor. For $\mathrm{T} \rightarrow 0$ thermal energy is exclusively in a boson field and not in an atomistic sub-system. Sufficiently strong but non-relevant atomistic sub-systems can increase the pre-factor of the universal power function of the heat capacity of the relevant boson field but they do not change the universal exponent $[6,27]$. On the other hand, at sufficiently high temperatures, thermal energy changes to the atomistic excitation systems because these systems have the lower dispersion energy. Nevertheless, because of the finite interactions between bosons and atomistic systems, the temperature dependence of the heat capacity assumes for all temperatures universality furnished from bosons.

It is evident that the heat capacity peak at the magnetic ordering temperature is dominated by the Goldstone bosons. Outside the critical range at $T_{c}$, the absolute values of the heat capacity are determined essentially by the non-magnetic inter- 
atomic interactions (phonons). According to the atomistic models of the lattice dynamics [33], these heat capacity values should be material specific throughout and should not exhibit universality. This, however, applies to the absolute heat capacity values only and not to the temperature dependence. On the other hand, it is very comfortable that in spite of complicated interatomic interactions and of complicated but quantized boson-phonon interactions, the temperature dependence of the observed heat capacity exhibits universality, though a nonintrinsic universality, irrespective of whether the bosons or the phonons give the dominant contribution to the heat capacity.

Since in the non-magnetic solids the wavevector dependence of the phonons gets modified by the Debye boson-phonon interaction, it can be expected that, according to the principle of reciprocity, the dispersion relations of the Debye bosons get correspondingly modified $[14,27,30]$. As we have shown recently $[27,30]$, the dispersion relations of the Debye bosons can easily be constructed for all energies up to a thermal energy that corresponds to the melting temperature $\left(\mathrm{T}_{\mathrm{m}}\right)$, using the known temperature dependence of the sound velocities, calculated from the elastic constants [35]. Since $T_{m}$ [36] is generally large than the Debye temperature $\Theta_{D}$ [37] it follows that the dispersion energy of the Debye bosons exceeds the dispersion energy of the phonons by far. Only for thermal energies of lower than $\mathrm{k}_{\mathrm{B}} \cdot \Theta_{\mathrm{D}}$ for which the phonons are partly excited only, the dispersion of the Debye bosons is a nearly linear function of wave-vector. When all phonons are excited, that is for energies of larger than $\mathrm{k}_{\mathrm{B}} \cdot \Theta_{\mathrm{D}}$, the dispersion of the Debye bosons becomes a power function of wave vector $\left(\sim q^{x}\right)$ with a rational exponent $x$ of smaller than unity $[27,30]$. An exponent of $x<1$ means a damped propagation of the Debye bosons. The discrete values of $x$ show that there are a few distinguished modes of boson-phonon interaction only. In other words, approximately at $\mathrm{k}_{\mathrm{B}} \cdot \Theta_{\mathrm{D}}$ an analytical crossover from a nearly linear dispersion at low energies to a power function of wave-vector at high-energies occurs in the dispersion relation of the Debye bosons [27]. Moreover, the dispersion of the Debye bosons exceeds the zone boundary. Since the sources of the Debye bosons are the atoms, the shortest possible wave-length of the Debye bosons is of the order of the diameter of the atoms and therefore is smaller than the lattice parameter. The largest wave-vector of the Debye bosons therefore is larger than the zone boundary $[27,30]$.

In the same way, we must assume that the dispersion relations of the Goldstone bosons get modified by the boson-magnon interaction, at least for thermal energies for which the density of states of the magnons is sufficiently high. Unfortunately, for higher excitation energies there is no quantitative information available as to the wavevector dependence of the Goldstone bosons. Note that mass-less bosons cannot be observed using inelastic neutron scattering. Only for low thermal energies for which magnons are weakly excited only, it could be evidenced experimentally that the dispersion relations of the Goldstone bosons, at the critical temperature $\mathrm{T}=0$, are simple power functions of wave-vector with rational exponents that are characteristic for the dimensionality of the global boson field [7,38]. Additionally, the exponents are different for magnets with an integer and with a half-integer spin [7]. The energy densities -or heat capacities- of the boson fields generated by the two spin species therefore are different. Note that the deviation of the spontaneous magnetization from saturation at $\mathrm{T}=0$ is given by the heat capacity of the Goldstone boson field at $\mathrm{T}=0$ and therefore is universal but is different for magnets with an integer and with a half-integer spin [5-7]. The spin dependence is a typical quantum effect associated with the fact that the number of states per spin is even for halfinteger spins but odd for integer spins. However, for the generation of magnetic dipole radiation by the precessing spins there is no quantitative theoretical description available as yet.

The aim of this communication is to demonstrate on account of quantitative but rather empirical analyses of published heat capacity data $[39,40]$ that in the magnetic solids the temperature dependence of the heat capacity is determined for all temperatures by the Goldstone bosons in a similar way as it is determined by the Debye bosons in the non-magnetic solids [27]. In contrast to the non-magnetic solids, evidence for the necessary interactions between the Goldstone bosons and the magnons is available from the magnon dispersion relations only but not from the dispersion of the Goldstone bosons that are known for low thermal energies only, where interaction effects with the magnons and/or phonons are below the threshold to induce an analytical crossover to a different wave-vector dependence $[7,38]$. 
A general result of our analyses is that on the high-temperature side of the magnetic ordering temperature, $\mathrm{T}_{\mathrm{c}}$, the critical heat capacity is divergent. According to the here adopted sign convention, divergence of the heat capacity is expressed by a negative exponent $\alpha$. Many of the observed $\alpha$-values for $\mathrm{T}>\mathrm{T}_{\mathrm{c}}$ fall in the sequence $\alpha=0,-1 / 4,-2 / 4,-3 / 4,-4 / 4$ and $-5 / 4$. Divergence of the heat capacity for $T>T_{c}$ is indicative of weak boson-phonon interactions. This conforms to the generally weak spontaneous magnetostriction for $\mathrm{T}>\mathrm{T}_{\mathrm{c}}$ [6]. On the low-temperature side of $\mathrm{T}_{\mathrm{c}}$ the critical heat capacity is finite. This is in accord with the rather strong spontaneous magnetostriction. A clearly identified sequences for the critical exponent $\alpha$ is $\alpha=1 / 2,1 / 3,1 / 4,1 / 5$ and $1 / 6$ but other exponents are observed in addition (see Chapter Results below). As we have mentioned, the interpretation of these exponents is difficult because too many parameters are involved.

Since for $T<T_{c}$ the heat capacity is finite at $T_{c}$ (the critical exponent $\alpha$ is positive according to our sign convention) but divergent for $\mathrm{T}>\mathrm{T}_{\mathrm{c}}$, the heat capacity is discontinuous at $\mathrm{T}_{\mathrm{c}}$. This result is in contrast to the atomistic theories [8] and to many earlier heat capacity analyses that made an explicit subtraction of an assumed non-magnetic heat capacity background [21-24]. It is evident that the as obtained $\alpha$-values depend critically on the assumed non-magnetic background.

Note that all our fit functions contain three fit parameters only: the critical exponent, the prefactor of the critical power function and an absolute constant. The critical temperature is fixed to the sharp maximum of the heat capacity. According to our aforementioned arguments, no explicit background is subtracted from the experimental heat capacity data. A constant background is automatically accounted for by the fitted absolute constant that includes magnetic and non-magnetic heat capacity contributions in an unclear way and, as a consequence, is difficult to interpret. The same applies to the fitted pre-factor of the critical power function. The only revealing fit parameter is the exponent $\alpha$. However, as we have mentioned, the actual value of $\alpha$ depends in an unclear way on the interaction between the Goldstone bosons with all other degrees of freedom. It is, however, considered as a proof of consistence for our fitting procedure that, within the experimental error limits, the critical exponents $\alpha$ come out as rational numbers that are reproduced by different materials.
On the other hand, we have to admit that it is not possible to verify a diverging heat capacity experimentally. The problem in reliable heat capacity measurements very near to $T_{c}$ is that for structurally perfect and pure single crystals, the heat capacity peak at $\mathrm{T}_{\mathrm{c}}$ can be extremely sharp. Since heat capacity measurements require the application of a finite heat pulse, the experimental temperature resolution is limited to the temperature increment induced by the heat pulse. In fact, a diverging heat capacity at $T_{c}$ can be rationalized by extrapolation only. This, however, is reasonably possible since the critical power functions hold over a finite distance from $T_{c}$, as it is well-known for the other thermodynamic observables such as the critical susceptibility, the spontaneous magnetization or the two-spin correlation length [5-7]. The only problem is to identify the upper and lower bounds of the fit range and to exclude all data beyond this range from the fit. Both limits are not very sharp and have to be made sure by appropriate variations of the fit rang. For temperatures sufficiently away from $T_{c}$ the critical range is limited by the crossover from the power function of the argument $\left|\mathrm{T}-\mathrm{T}_{\mathrm{c}}\right|$ to a power function of absolute temperature. On the other hand, very near to $T_{c}$ the limit of the fit range depends on the experimental conditions and on the structural perfection of the sample, and is more difficult to specify generally. Due to the inevitably limited temperature resolution, the heat capacity measurements very near to $T_{c}$ integrate over the narrow heat capacity peak and let the heat capacity appear finite at $T_{c}$ all the more the heat capacity is finite for $T \leq T_{c}$ [21-24]. Moreover, the two heat capacity branches for $\mathrm{T}<\mathrm{T}_{\mathrm{c}}$ and for $\mathrm{T}>\mathrm{T}_{\mathrm{c}}$ seem to approach each other for $\mathrm{T} \rightarrow \mathrm{T}_{\mathrm{c}}$. This artefact is due to the limited temperature resolution and suggests a continuous behavior of the heat capacity at $T_{c}$ with positive values of $\alpha$ on both sides of $T_{c}$ (according to our sign convention). At first-order transitions with a discontinuous spontaneous magnetization the heat capacity can be divergent on both sides of $\mathrm{T}_{\mathrm{c}}$.

As we have already mentioned, outside the critical range at $\mathrm{T}_{\mathrm{c}}$, the heat capacity exhibits also universality and can sectionally be described by universal power functions of absolute temperature according to the expression $\overline{c_{p}(T)=A}+B \cdot T^{\varepsilon}$. Two variants of this expression have to be distinguished. At high-temperatures, commonly for $T>T_{c}$, the coefficient $A$ is positive but $B$ and the exponent $\varepsilon$ are negative. Formally, this variant of $c_{p}(T)$ describes the deviations of the heat 
capacity from the D-P limit. However, as for the non-magnetic solids, the fitted value for the asymptotic parameter $\mathrm{A}$ is generally larger than the theoretical D-P value, that is given by $3 n \cdot N_{L} \cdot k_{B}$, with $n$ as the number of atoms per formula unit, $N_{L}$ the number of atoms per mole and $\mathrm{k}_{\mathrm{B}}$ as the Boltzmann constant. On the other hand, as for all fitted absolute constants, the fit result for the asymptotic parameter A seems to be of no physical significance since before saturation at $\mathrm{c}_{\mathrm{p}}=\mathrm{A}$ is reached, a crossover occurs, rather precisely at the D-P limit, i.e. at $\mathrm{T} \sim \Theta_{\mathrm{D}}$, where the heat capacity deflects suddenly from the function $c_{p}(T)=A+B \cdot T^{\varepsilon}$ towards larger values [27,41]. Note that heat capacity values of larger than the D-P limit are beyond the atomistic concepts and have to be attributed to the bosons that drive the solid-liquid phase transition [41]. Most of the observed exponents $\varepsilon$ fall in the sequence $\varepsilon=0,-2 / 4,-3 / 4$, $4 / 4,-5 / 4,-6 / 4$. The same rational exponent values occur also for the critical exponents $\alpha$ for $\mathrm{T}>\mathrm{T}_{\mathrm{c}}$, but not necessarily at the same material. Nevertheless, the agreement of the $\varepsilon$-values with the typical magnetic exponent $\alpha$ provides some evidence that the exponents $\varepsilon$ are due to the Goldstone bosons and not due to the Debye bosons. Moreover, most of the exponents $\varepsilon$ observed in the magnetic solids are different compared to the corresponding exponents in the non-magnetic solids that, commonly, are $\varepsilon^{=-1},-4 / 3$ and $-5 / 4$ [27]. Many of the exponent $\alpha$ on the low-temperature side of $T_{c}$ can be grouped according to the sequences $\alpha=1 / 2$, $1 / 3,1 / 4,1 / 5$ and $1 / 6$. Alternatively, the exponents can be grouped according to the sequence $\varepsilon=2 / 3$, $2 / 4,2 / 5$ and $2 / 6$, while $\alpha=1$ seems to be forbidden.

At low-temperatures, several temperature sections with different functions of type $c_{p}(T)=A+B \cdot T^{\varepsilon}$ can be distinguished. Common to these functions is that the coefficient $B$ and the exponent $\varepsilon$ are positive, while $\mathrm{A}$ is negative. The observed exponents can be grouped into the two sequences $\varepsilon=1 / 2,2 / 2,3 / 2,4 / 2,5 / 2,6 / 2$ and $\varepsilon=1 / 3$, $3 / 3,4 / 3$. Only for the asymptotic $T^{3}$ function according to Debye, the absolute constant $\mathrm{A}$ is zero. In other words, a finite absolute constant, A, indicates that the power function is not the asymptotic behavior for $\mathrm{T} \rightarrow 0$. In the following chapter we start the discussion with the temperature dependence of the heat capacity for $\mathrm{T}<\mathrm{T}_{\mathrm{c}}$ for magnetic materials with a rather high magnetic ordering temperature.

\section{The Heat Capacity for $T<T_{c}$}

The most critical point in fitting a power function of temperature to a particular temperature section of the heat capacity is the identification of the upper and lower bounds of this function. As we have already mentioned, the validity range of each power function of temperature is limited by the crossover events to the adjacent power functions. However, the fit cannot be conducted just to the crossover temperature since a crossover is not a very sharp event but can be considerably rounded. The rounded region has to be excluded from the fit. Since we can be rather sure that all critical exponents are rational numbers, it is useful to plot, in a first step, the heat capacity data on a $\mathrm{T}^{\varepsilon}$ temperature scale, using suitable rational test values for $\varepsilon$, and looking for a section with a linear dependence on the $\mathrm{T}^{\varepsilon}$ scale. When the validity limits of the $\mathrm{T}^{\varepsilon}$ function are identified, the exponent can be fitted reliably.

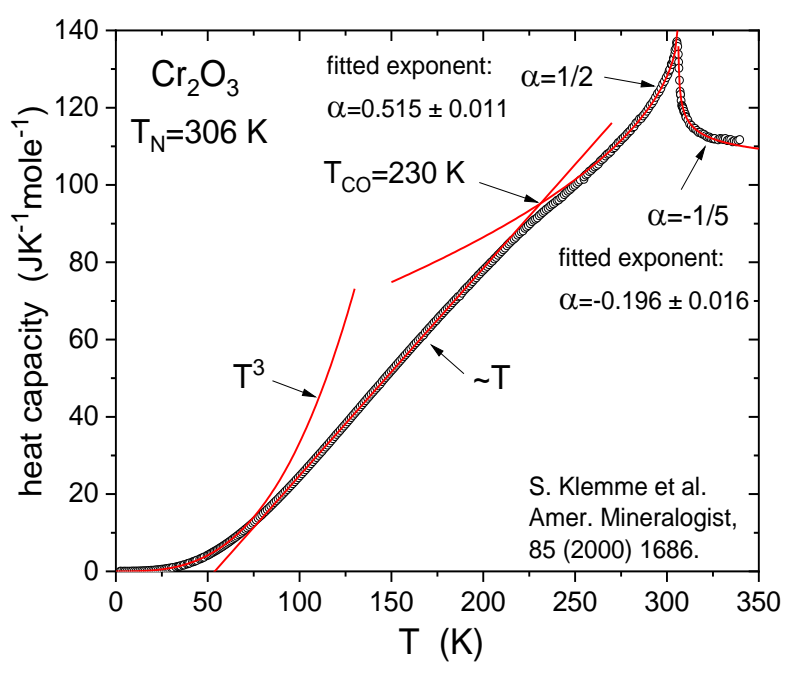

Figure 1. In the temperature range shown, the heat capacity of the rhombohedral antiferromagnet $\mathrm{Cr}_{2} \mathrm{O}_{3}$ can be described by four universal power functions of temperature [42]. Note that the theoretical Dulong-Petit limit $\left(124.75 \mathrm{JK}^{-1}\right.$ mole $\left.^{-1}\right)$ is reached for higher temperatures than are shown. The exponent of the asymptotic power function for $T \rightarrow \infty$ is $\varepsilon=-2 / 3$ (not shown) [36].

Figure 1 shows the fit results for the heat capacity of the rhombohedral antiferromagnet $\mathrm{Cr}_{2} \mathrm{O}_{3}$ [42]. For the temperature range shown, four sections with different power functions of temperature can be distinguished. One further temperature power function can be expected for $\mathrm{T} \rightarrow \infty$. Since $\mathrm{Cr}_{2} \mathrm{O}_{3}$ is a hard material with a high Debye temperature, the heat capacity approaches the D-P limit for $\mathrm{T} \rightarrow \infty$ for higher temperatures than in Figure 1. From the high-temperature data 
of [36] an exponent of $\varepsilon=-2 / 3$ (fitted exponent: $\varepsilon=-$ $0.688 \pm 0.107)$ can be inferred. It should be noted that over the temperature range of the Debye $\mathrm{T}^{3}$ function and of the following $\sim \mathrm{T}$ function, the spontaneous sublattice magnetization decreases by a single $\mathrm{T}^{3}$ function $[6,43]$. The $\mathrm{T}^{3}$ function in the spontaneous magnetization is characteristic for the heat capacity of the 1d-Goldstone boson field at the critical temperature $\mathrm{T}=0$ in magnets with an integer spin [6,7]. The one-dimensional dynamic symmetry conforms to the axial lattice structure of rhombohedral $\mathrm{Cr}_{2} \mathrm{O}_{3}$. In other words, there is only one domain type with all spins oriented along the rhombohedral c-axis $[43,44]$.

Two power functions in the heat capacity over a temperature range over which the spontaneous magnetization is given by a single $\mathrm{T}^{3}$ function illustrates that different energy degrees of freedom dominate the heat capacity at different temperatures. Note that for magnets with a high ordering temperature the magnetic contributions to the low-temperature heat capacity are negligible such that the Debye bosons dominate the heat capacity. If the $\mathrm{T}^{3}$ function holds without absolute constant, all thermal energy is in the Debye boson field. From the pre-factor of the asymptotic $\mathrm{T}^{3}$ function, a Debye temperature of $\Theta_{D}=663 \mathrm{~K}$ can be calculated for $\mathrm{Cr}_{2} \mathrm{O}_{3}$. Another situation, allowing for a clear interpretation of the lowtemperature heat capacity data is given for the magnets with a very low ordering temperature (see discussion of Figure 12 below). In this case the magnetic degrees of freedom dominate the heat capacity for $\mathrm{T} \rightarrow 0$ and the contribution of the Debye bosons is negligible. All thermal energy then is in the Goldstone boson field, and the heat capacity for $T \rightarrow 0$ is given by the $T^{\varepsilon}$ function of the heat capacity of the Goldstone boson field. This $\mathrm{T}^{\varepsilon}$ function defines the thermal decrease of the spontaneous magnetization.

Since the $\mathrm{T}^{3}$ function in the thermal decrease of the spontaneous magnetization is indicative of an integer spin but, according to Hund's rules, the free $\mathrm{Cr}^{3+}$ has a half-integer spin of $\mathrm{S}=3 / 2$, it follows that the crystal field interaction is relevant in $\mathrm{Cr}_{2} \mathrm{O}_{3}[6,45]$. The crystal electric field is another example illustrating that the boson-controlled dynamics reacts in a discrete and threshold induced way on continuous variations of atomistic quantities, such as the crystal electric field. Only a sufficiently strong crystal field can become relevant, i.e. can induce a crossover to another universality class of the spontaneous magnetization. A weaker crystal field interaction has no effect $[6,45]$. Because in the $3 d$-metal compounds the orbital moments of the $3 \mathrm{~d}$ ions are largely quenched, magnetism is manly of spin type and crystal field interaction is relatively weak. Note that the crystal electric field acts on the orbital moment. In the boson controlled ordered state of the 3d-metal compounds, the common effect of a relevant crystal field is to remove only one of the $2 \mathrm{~S}+1$ spin-states from the dynamics [45]. This means that the spin of the $\mathrm{Cr}^{3+}$ ion in $\mathrm{Cr}_{2} \mathrm{O}_{3}$ is reduced from $\mathrm{S}=3 / 2$ to $\mathrm{S}_{\text {eff }}=1$. In this way the dynamic universality class of the spontaneous magnetization changes from $T^{5 / 2}$ for $S=3 / 2$ to $T^{3}$ for $S_{\text {eff }}=1 \quad[6,7]$. Consistent with a reduced effective spin is a reduced saturation magnetic moment [44]. Note, however, that in non-cubic crystals, the dimensionality of the global boson field can depend on sample preparation [48]. In axial crystals the boson field needs not be onedimensional. This is a question of the domain configuration. Large single crystals can be considerably strained due to the crystal grows process. Strain can change the configuration of the magnetic domains and therefore the dimensionality of the global boson field.

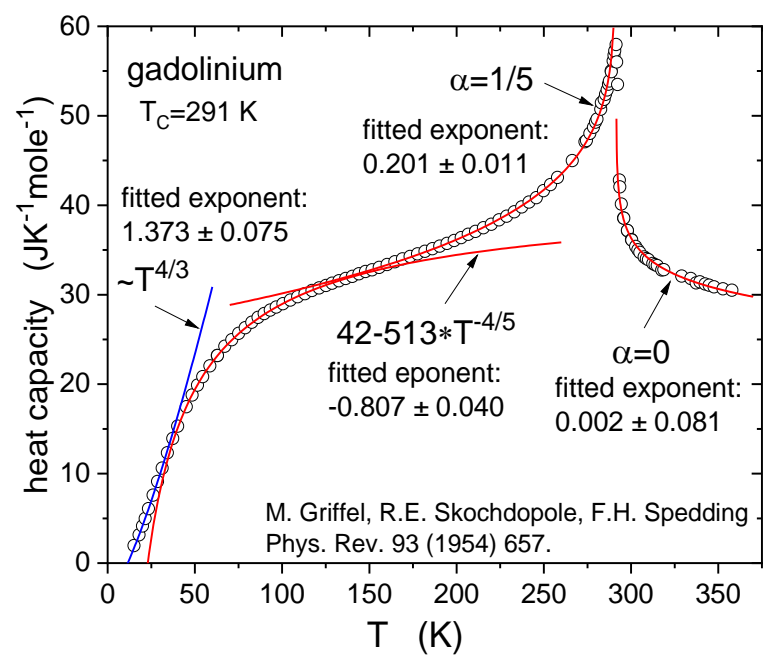

Figure 2. In the temperature range shown, the heat capacity of ferromagnetic gadolinium with hexagonal hcp-structure and $S=7 / 2$ can be fitted by four universal power functions of temperature [46]. Note the unusually large critical range for $T<T_{C}$. For $T>T_{C}$ the critical heat capacity is logarithmically divergent.

The crossover temperature at $\mathrm{T}_{\mathrm{CO}}=230 \mathrm{~K}$ from the $\sim \mathrm{T}$ function to the critical power function with $\alpha=1 / 2$ nicely agrees with the crossover temperature from $\mathrm{T}^{3}$ function to the critical power function with $\beta=1 / 3$ in the spontaneous sublattice- 
magnetization of $\mathrm{Cr}_{2} \mathrm{O}_{3}$ [6]. Since, except for the Debye $\mathrm{T}^{3}$ function, the fitted power functions in Figure 1 are supposed to be induced by the Goldstone boson field, the observed universal exponents can be expected to be independent of the spin structure but they are likely to depend on whether the spin quantum number is integer or half-integer. This, however, is difficult to evidence in general since the spin quantum number can be ambiguous through the action of a relevant crystal field [45].

For mechanically soft materials with a low Debye temperature the asymptotic function for $\mathrm{T} \rightarrow \infty, \mathrm{c}_{\mathrm{p}}(\mathrm{T})=\mathrm{A}+\mathrm{B} \cdot \mathrm{T}^{\varepsilon}$ (with $\mathrm{B}$ and $\varepsilon$ negative), sets in at a fairly low temperature. For the metallic, hexagonal ferromagnet gadolinium, the Debye temperature of $\Theta_{D}=182 \mathrm{~K}$ [37] is considerably lower than the Curie temperature of $\mathrm{T}_{\mathrm{C}}=291 \mathrm{~K}$ [47]. As a consequence, the asymptotic $\mathrm{c}_{\mathrm{p}}(\mathrm{T})=\mathrm{A}+\mathrm{B} \cdot \mathrm{T}^{\varepsilon}$ function, with $\mathrm{B}$ and $\varepsilon$ negative, appears already on the low-temperature side of the heat capacity peak at $\mathrm{T}_{\mathrm{C}}$. Due to the large spin quantum number of $S=7 / 2$, the magnetic contribution to the total heat capacity of the ordered state is unusually large. In fact, for $\mathrm{T}>75$ $\mathrm{K}$ the heat capacity values in Figure 2 are already beyond the theoretical D-P limit of $24.95 \mathrm{JK}^{-}$ ${ }^{1} \mathrm{~mole}^{-1}$. As it is generally observed, the fitted value for the asymptotic heat capacity $(T \rightarrow \infty)$ of $A=42$ $\mathrm{JK}^{-1}$ mole $^{-1}$ is much larger than the theoretical D-P limit and has an unclear physical significance. The enormous large critical range on the lowtemperature side of $\mathrm{T}_{\mathrm{C}}$ with critical exponent of $\alpha=1 / 5$ conforms to the large critical range of the spontaneous magnetization with critical exponent of $\beta=1 / 3[6,47]$. Note, however, that in non-cubic magnets, the critical exponent $\beta$ can depend on the shape and size of the sample when the mean free path of the bosons is of the order of the linear dimensions of the sample $[32,48]$. For a long-stick single crystal, cut parallel to the c-axis, the critical behavior is in favor of a one-dimensional dynamic symmetry, and $\beta=1 / 3$ is observed $[6,47]$. On the other hand, for a small spherical sample with a diameter of smaller than the mean free path of the Goldstone bosons, all bosons get reflected on the inner surface of the sample. The resulting boson field and the dynamic symmetry then are $3 \mathrm{~d}$ isotropic. For a half-integer spin the critical exponent $\beta$ then is $\beta=1 / 2[6,32]$. We therefore have to assume that the fitted exponents in Figure 2 are not generally specific for Gd but can depend on the size and shape of the investigated sample.
In the nominally cubic antiferromagnet $\mathrm{CoO}$ spontaneous tetragonal lattice distortions set in at the Néel temperature [28,29]. Depending on sample preparation, crystal field effects can become additionally relevant [6]. This makes the interpretation of the fitted exponents for the heat capacity in Figure 3 ambiguous. Tetragonal lattice distortions have been observed for $\mathrm{CoO}$ powder material using neutron scattering [28] and synchrotron radiation diffraction [29]. However, it can depend on the grain size and on the quality of the powder material whether lattice distortions are sufficiently strong to induce a crossover to a lower dimensionality of the global boson field [48]. Commonly, small powder grains are less distorted than large single crystals. If the crystal field interaction is not relevant, the spin of the $\mathrm{Co}^{2+}$ ion is $\mathrm{S}=3 / 2$ and thermal decrease of the spontaneous magnetization of the undistorted $\mathrm{CoO}$ crystal is expected to be by $\mathrm{T}^{2}$ function [6,7]. For larger single crystals lattice distortions commonly are large. For a relevant tetragonal (axial) lattice distortion, thermal decrease of the spontaneous magnetization is by $\mathrm{T}^{5 / 2}$ function for an halfinteger spin of $S=3 / 2$ [6]. On the other hand, for commercially available powder material the $T^{9 / 2}$ function of the isotropic boson field in magnets with an integer spin has been observed [6]. As a consequence, the powder grains of this sample are weakly strained only. According to a measured saturation magnetic moment for this $\mathrm{CoO}$ powder material of $\mathrm{m}_{\mathrm{s}}=3.98 \pm 0.06 \mu_{\mathrm{B}} / \mathrm{Co}$ the spin cannot be $S_{\text {eff }}=1$ but must be $S_{\text {eff }}=2$ [29]. In other words, it is possible that the orbital degrees of freedom add one quantum state to the $2 \mathrm{~S}+1$ spin states. The g-factor then falls below $\mathrm{g}=2$ [45].

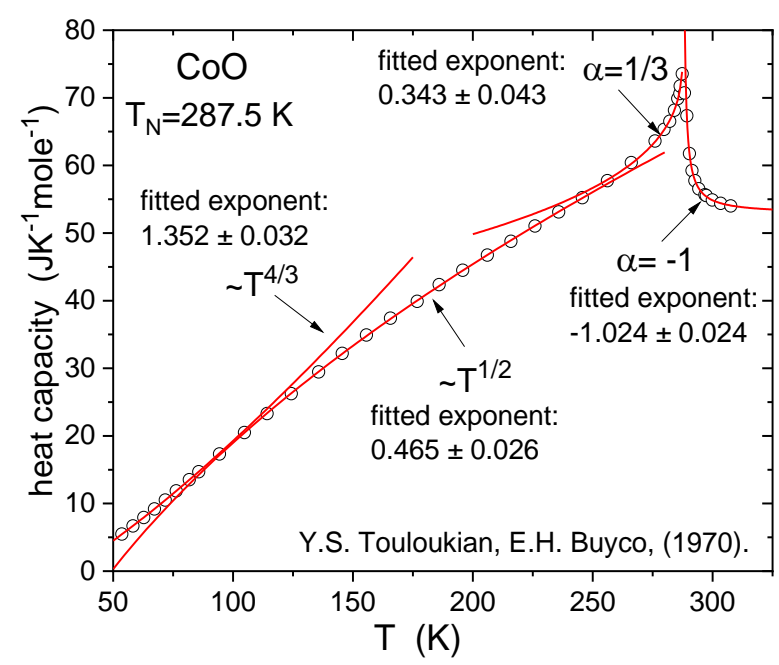

Figure 3. For the temperature range shown, the heat capacity of antiferromagnetic $\mathrm{CoO}$ can be 
described by four universal power functions of temperature [39].

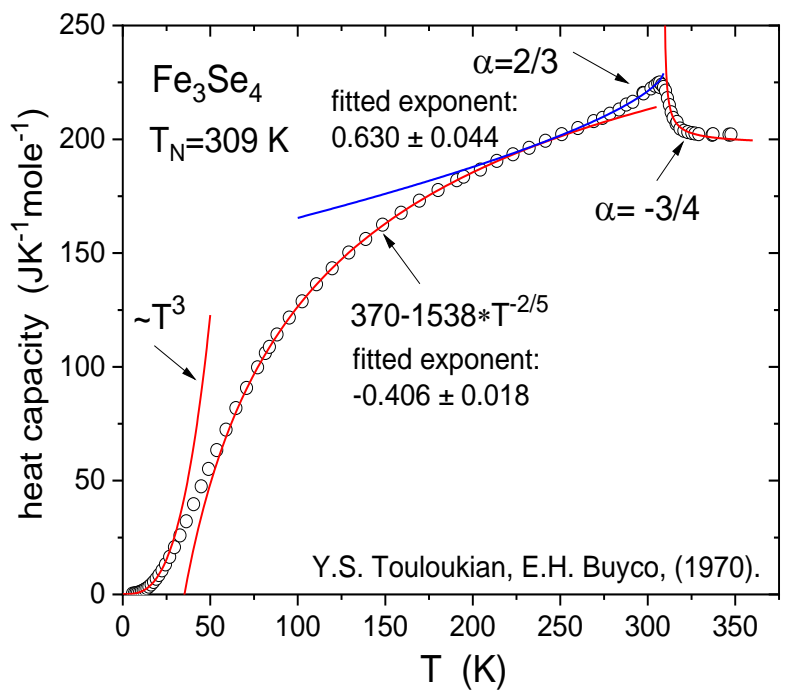

Figure 4. For monoclinic $\mathrm{Fe}_{3} \mathrm{Se}_{4}$ with a Néel temperature of as high as $T_{N}=309 \mathrm{~K}$ and a Debye temperature of only $\Theta_{D}=126 \mathrm{~K}$ the asymptotic power function for $T \rightarrow \infty, c_{p}(T)=A+B \cdot T^{\varepsilon}$, with negative exponent and negative pre-factor, $B$, appears on the low temperature side of $T_{N}$ [39]. Note that the theoretical Dulong-Petit limiting value is $c(D-P)=174.7 \mathrm{JK}^{-1}$ mole $^{-1}$.

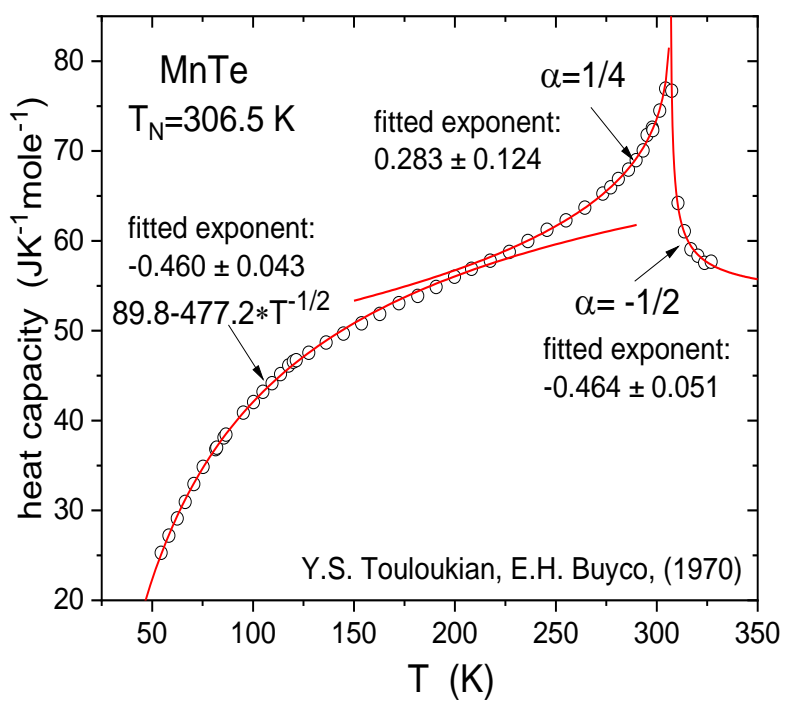

Figure 5. In hexagonal MnTe the asymptotic power function for $T \rightarrow \infty, c_{p}(T)=A+B \cdot T^{\varepsilon}$, with negative pre-factor $B$, and negative exponent $\varepsilon$ appears on the low-temperature side of $T_{N}=306.5$ $K$ [39] as it is typical for materials with $\Theta_{D}<T_{N}$.

Since in monoclinic $\mathrm{Fe}_{3} \mathrm{Se}_{4}$ the asymptotic heat capacity for $\mathrm{T} \rightarrow \infty$ appears at relatively low temperatures, the Debye temperature must be low
(Figure 4). In fact, from the fitted low-temperature $\mathrm{T}^{3}$ function a Debye temperature of $\Theta_{\mathrm{D}}=126 \mathrm{~K}$ can be calculated. A similar behavior is observed for MnTe (Figure 5).

In nominally cubic $\mathrm{NiO}$, spontaneous rhombohedral lattice distortions set in at the Néel temperature [28]. Consistent with an integer spin of $\mathrm{S}=1$ of the $\mathrm{Ni}^{2+}$ ion and with a relevant axial lattice distortion is a thermal decrease of the spontaneous magnetization according to a $\mathrm{T}^{3}$ function [6]. For this universality class, the bosonmagnon interaction is particularly strong, giving rise to a large magnon excitation gap [6]. For NiO the gap is $~ 4.54 \mathrm{meV}$ (compare discussion of Figure 19) [6,49]. In Figure 6 four power functions of temperature are fitted to the heat capacity of $\mathrm{NiO}$ [39]. For $\mathrm{NiO}$ with a Néel temperature of as high as $\mathrm{T}_{\mathrm{N}}=523 \mathrm{~K}$ the magnetic contributions to the low-temperature heat capacity are negligible and the heat capacity is given by the Debye $\mathrm{T}^{3}$ function. Note, however, that two $\mathrm{T}^{3}$ functions with different pre-factors have to be distinguished $[6,27]$. The non-asymptotic $\mathrm{T}^{3}$ function has a larger pre-factor and includes a small negative absolute constant. The larger pre-factor is due to the finite heat capacity contributions of the nonrelevant phonons. In Figure 6 the non-asymptotic $\mathrm{T}^{3}$ function is depicted. From the asymptotic $\mathrm{T}^{3}$ function (with no absolute constant) a Debye temperature of $\Theta_{D}=610 \mathrm{~K}$ is obtained. As a consequence, the heat capacity approaches the D$\mathrm{P}$ limit for temperatures outside the temperature window of Figure 6 (see Figure 19).

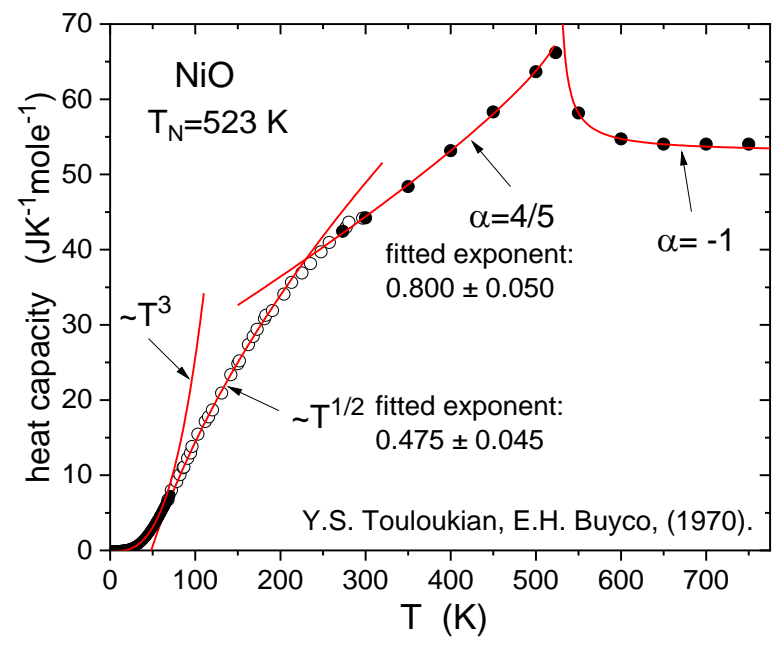

Figure 6. Heat capacity of $\mathrm{NiO}$ combined from two literature sources [39]. The spin of the $\mathrm{Ni}^{2+}$ ion is $S=1$. For $T \leq T_{N}$ the cubic lattice is rhombohedrally distorted [28]. The low-temperature $T^{3}$ data are from this work (see text). 


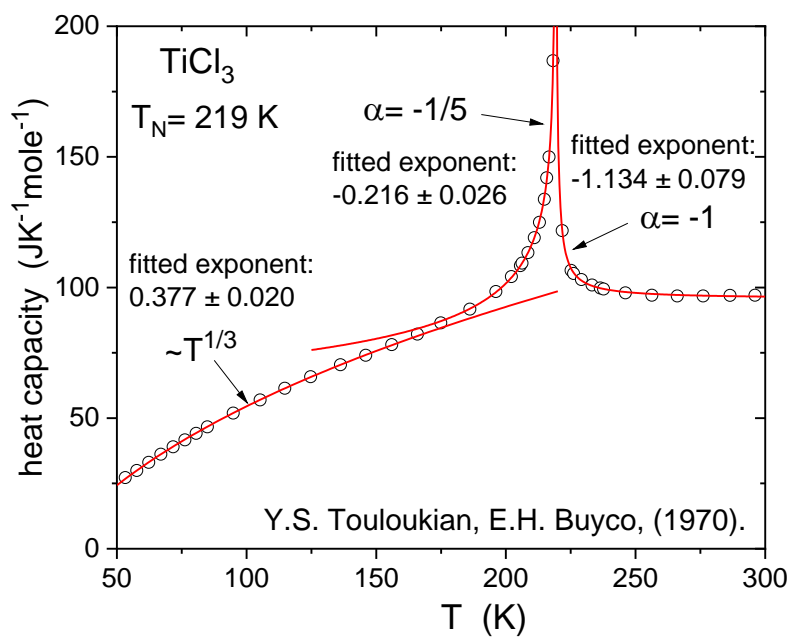

Figure 7. Heat capacity of monoclinic $\mathrm{TiCl}_{3}$ with $S=1 / 2$ due to the $\mathrm{Ti}^{3+}$ ion, as a function of absolute temperature [39]. The negative exponent $\alpha$ on the low-temperature side of $T_{N}$ is indicative of a firstorder magnetic phase transition. Because of the low spin moment, the magnetic contribution to the heat capacity is low and the theoretical DulongPetit limit of $c(D-P)=99.7 \quad J^{-1} m^{-1}$ is reasonably realized.

\section{The Critical Heat Capacity at $T_{c}$}

In the past, the ferromagnets $\mathrm{EuS}$ and $\mathrm{EuO}$ and the antiferromagnets EuTe and $\mathrm{RbMnF}_{3}$ were considered as nearly ideal realizations of the Heisenberg model. In fact, all four materials have cubic lattice structure and pure spin moments. However, as we have explained, in contrast to the assumptions of the Heisenberg model, the ordering transitions of magnets with a three-dimensional spin are not driven by the exchange interactions between the spins but by the Goldstone boson field. Evidence for boson dynamics is provided by the finite width of the critical range and by the rational critical exponents $[5,6]$. By the way, the finite width of the critical range is condition for a reliable experimental evaluation of the critical exponents. Note that the critical exponents predicted by the atomistic models pertain to the leading term of a power series expansion at the critical point $[8,17,18]$. Power series contain too many fit parameters and can nearly not be verified experimentally. The measured critical exponents of the four mentioned materials agree, in fact, within the experimental error limits $(\beta=1 / 3, \gamma=4 / 3$ and $v=2 / 3)[5,15,50]$. Independence of the critical exponents on the spin structure proves impressively that the exchange interactions between the spins are excluded from the critical dynamics, i.e. are not relevant.
However, there is an intriguing particularity with these critical exponents. This becomes obvious by a comparison with tetragonal $\mathrm{MnF}_{2}$ [51] that is, perhaps, the best investigated bosoncontrolled one-dimensional antiferromagnet: the critical exponents of EuS, EuO, EuTe and $\mathrm{RbMnF}_{3}$ agree within error limits with those of the monodomain antiferromagnet $\mathrm{MnF}_{2}$ [15]. As a conclusion, in spite of their cubic lattice structures the ordering transitions of EuS, EuO, EuTe and $\mathrm{RbMnF}_{3}$ are driven, as for tetragonal $\mathrm{MnF}_{2}$, by a one-dimensional boson field. This can easily be explained, assuming that, for some reasons, in the four cubic materials the boson fields of the domains long $\mathrm{x}-, \mathrm{y}-$ and $\mathrm{z}$-axis are not coupled dynamically [15]. For instance, when the domains are rather large, the boson fields of adjacent domains are not in dense contact and interaction between them becomes less likely. For completely decoupled domains, the observed critical behavior of the bulk material is determined by the behavior of the isolated magnetic domain, and is onedimensional. The is as for bulk $\mathrm{MnF}_{2}$ that can be considered as one large magnetic domain [51]. All $\mathrm{Mn}^{2+}$ moments are rigidly coupled to the onedimensional boson field along the tetragonal caxis. Typical for the one-dimensional dynamic symmetry of $\mathrm{MnF}_{2}$ is, that the two-spin correlation length and the staggered susceptibility transverse to the tetragonal c-axis, do not diverge at the Néel temperature [51]. For cubic magnets with a halfinteger spin, for which the domains along $x^{-}, y_{-}$ and $\mathrm{z}$-axis are coupled dynamically, the global boson field is $3 \mathrm{~d}$, and the critical behavior is of mean field type [5,6]. Famous examples of mean field critical behavior are the cubic ferromagnets $\mathrm{GdZn}$ and GdMg with $\mathrm{S}=7 / 2$ [6]. The important difference to the classical mean field approximation is that for the boson-defined meanfield critical behavior, the width of the critical range is finite and clearly limited by a crossover event. Moreover, for the cubic ferromagnet GdZn with $S=7 / 2$, discussed in [6], indications are obtained that the critical exponent is $\alpha=0$ (logarithmically divergent) on both sides of $\mathrm{T}_{\mathrm{C}}$ and therefore is at variance with the Landau model that predicts a finite heat capacity at $T_{c}$. Note that for the magnets with mean field critical behavior ( $\mathrm{S}=$ half-integer, $\mathrm{d}=3$ ) the interaction between bosons and magnons is very weak, as becomes evident by a very small magnon gap [5]. The boson magnon interaction seems to be decisive for the type of critical behavior [5]. 
In spite of identical critical exponents of $\beta=1 / 3$, $\gamma=4 / 3$ and $\nu=2 / 3$ the exponents $\alpha$ observed for the critical heat capacity of $\mathrm{EuS}, \mathrm{EuO}$, EuTe and $\mathrm{RbMnF}_{3}$ are different. One reason for this could be the different ordering temperatures and, as a consequence, the different proportions of magnetic to non-magnetic energy contributions at $\mathrm{T}_{\mathrm{c}}$. Additionally, the coupling strength between the magnetic and the elastic degrees of freedom will be different.

Figures 8-11 show the critical heat capacities of $\mathrm{EuS}$ [52], EuTe [23], EuO [22] and $\mathrm{RbMnF}_{3}$ [21] as a function of the temperature difference to the critical point. It can be seen, that in contrast to the identical critical exponents $\beta, \gamma$ and $v$, the fitted values for the critical exponents $\alpha$ are considerably different for the four materials and lack a clear systematic. Only for the critical exponent $\alpha$ for $\mathrm{T}<\mathrm{T}_{\mathrm{c}}$ of EuO, EuS and EuTe some correlation with the strength of the magnetostriction is possible, that is largest for $\mathrm{EuO}(\alpha=1 / 3)$, intermediate for $\operatorname{EuS}(\alpha=1 / 4)$ and very weak for EuTe $(\alpha=1 / 10)$ [53].

As Figures 8-11 show, in contrast to the method employed in the data analyses of [21-24], the critical exponents $\alpha$ obtained by our threeparameter fit never result in identical exponents above and below $T_{c}$. In particular, for $T>T_{c}$ the heat capacity turns out to be always divergent but finite for $\mathrm{T}<\mathrm{T}_{\mathrm{c}}$. With the exception of EuTe, $\alpha=0$ (logarithmic divergence) holds for $\mathrm{T}>\mathrm{T}_{\mathrm{c}}$. Formally, $\alpha=0$ fulfills the classical scaling relation $\alpha+2 \beta+\gamma=2$ either with $\beta=1 / 3$ and $\gamma=4 / 3$ for the magnets with 1d-boson field and half-integer spin, but also for the mean field critical exponents $\beta=1 / 2$ and $\gamma=1$ for the magnets with $3 \mathrm{~d}$-boson field and half-integer spin [7].

For EuS, the two available experimental data sets for the critical heat capacity are, practically, identical $[24,52]$. However, in the two publications the data were analyzed differently, and completely different values for the critical exponent $\alpha$ were obtained. In [52], the here favored three-parameter fit was made. Our fit results for the data of [52], shown in Figure 8, confirm the exponent values given in [52], i.e. $\alpha=0$ for $T>T_{C}$ and $\alpha=1 / 4$ for $\mathrm{T}<\mathrm{T}_{\mathrm{C}}$.

On the other hand, in [24] an explicit temperature dependent, non-magnetic heat capacity background was included in the fit, whereby the number of fit parameters is increased to four. Moreover, the fit was made using the constraint of identical critical exponents above and below $\mathrm{T}_{\mathrm{C}}$. This premise was considered as firmly approved by the available atomistic models, that are, however, inapplicable, since the dynamics is boson controlled. Under these assumptions, a finite heat capacity at $T_{C}$ and a non-rational critical exponent of $\alpha=0.133 \pm 0.003$ was obtained for EuS.

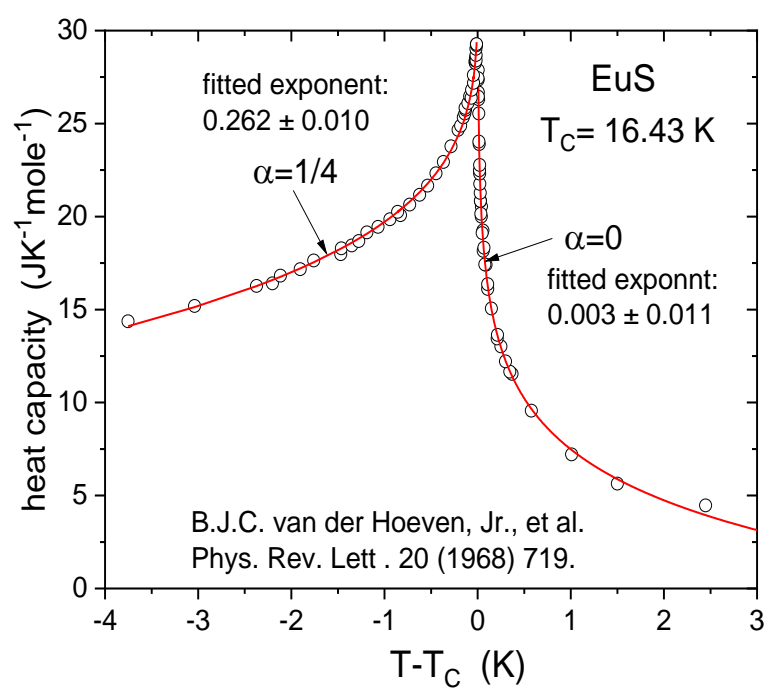

Figure 8. Critical heat capacity of the cubic ferromagnet EuS as a function of the temperature difference to the critical point [52]. For $T>T_{C}$ the heat capacity is logarithmically divergent $(\alpha=0)$. For $T<T_{C}$ the heat capacity is finite at $T_{C}$ $(\alpha=+1 / 4)$. The two, here fitted critical exponent values result from a three-parameter fit and agree with those given in [52] (see text).

In [23], the critical heat capacity data of antiferromagnetic EuTe were analyzed using the same four-parameter fit and the constraint of identical exponents above and below $\mathrm{T}_{\mathrm{N}}$ as in [24] for EuS. In contrast to EuS [24], a diverging heat with a critical exponent of $\alpha=-0.38 \pm 0.01$ was obtained for EuTe. Figure 9 shows our alternative analysis of the heat capacity data of [23] using the three-parameter fit as for EuS in Figure 8. It turns out that only for $\mathrm{T}>\mathrm{T}_{\mathrm{N}}$ the heat capacity of EuTe diverges with a rational critical exponent of $\alpha=$ $1 / 4$, for $T<T_{N}$ the heat capacity is finite $(\alpha=+1 / 10)$. The small value of $\alpha$ indicates some tendency for a first-order transition.

Critical heat capacity data of EuO [22] were fitted by the same four-parameter fit as for EuS [24] using the constraint of identical critical exponents $\alpha$ above and below $\mathrm{T}_{\mathrm{C}}$. In this way, the heat capacity came out to be finite at $\mathrm{T}_{\mathrm{C}}$ with a fitted critical exponent of $\alpha=0.044 \pm 0.01$. As the results of our three-parameter fit in Figure 10 show, the heat capacity of EuO is divergent $(\alpha=0)$ for $\mathrm{T}>\mathrm{T}_{\mathrm{C}}$. For $\mathrm{T}<\mathrm{T}_{\mathrm{C}}$ the heat capacity remains 
finite at $T_{C}$, and the critical exponent is very precisely $\alpha=1 / 3$.

The results of our three-parameter fit to the critical heat capacity data of $\mathrm{RbMnF}_{3}$ of [21] shown in Figure 11 are again in contrast to the four parameter fit of [21] that used the constraint of identical exponents $\alpha$ above and below $\mathrm{T}_{\mathrm{N}}$. The method of analysis of [21] resulted in a finite heat capacity above and below $T_{N}$ with a positive critical exponent of $\alpha=+0.14 \pm 0.01$, according to our sign convention. Note that a finite heat capacity for $\mathrm{T}>\mathrm{T}_{\mathrm{N}}$ can result when the too small experimental heat capacity data very close to $T_{N}$ are not excluded from the fit.

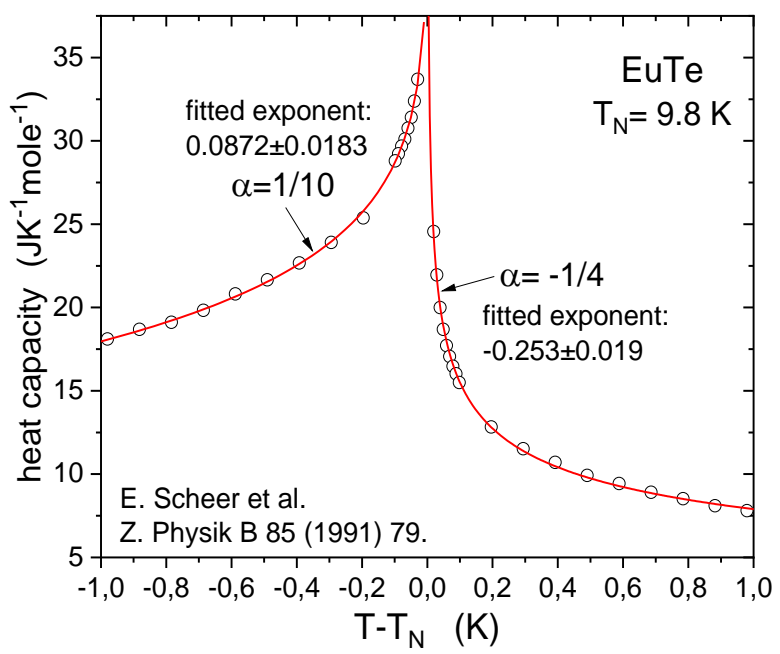

Figure 9. Critical heat capacity of antiferromagnetic EuTe as a function of the distance from Néel temperature [23]. Note the surprisingly different exponents compared to EuS (Figure 8). Using our three-parameter fit, with no explicit temperature dependence of a nonmagnetic heat capacity background, the heat capacity turns out to be divergent for $T>T_{N}$ only.

For the magnets with ordering temperatures of a few Kelvin only, the magnetic heat capacity for $\mathrm{T} \rightarrow 0$ is much larger than the non-magnetic heat capacity background due to the Debye bosons. One therefore might expect to observe the intrinsic critical behavior due the magnetic degrees of freedom. This, however, is not confirmed. Only the exponent $\varepsilon$ of the asymptotic $\mathrm{T}^{\varepsilon}$ power function in the heat capacity for $\mathrm{T} \rightarrow 0$ agrees with the $T^{\varepsilon}$ power function in the thermal decrease of the spontaneous magnetization and therefore is intrinsically magnetic [6]. This proves that the thermal decrease of the spontaneous magnetization with respect to saturation at $T=0$ is given by the heat capacity of the Goldstone boson field at $\mathrm{T}=0$.

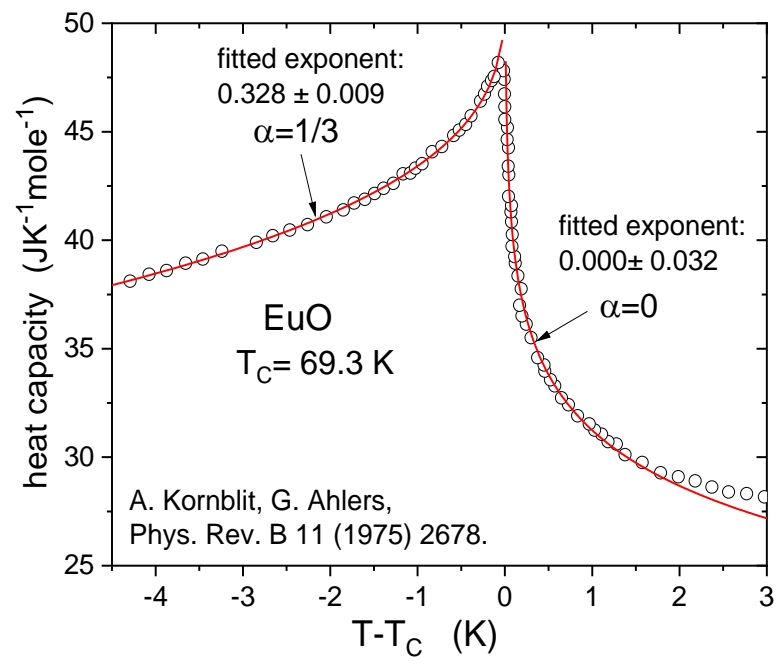

Figure 10. Critical heat capacity of EuO as a function of the distance from the critical temperature [22]. As for EuS (Figure 8) the heat capacity is logarithmically divergent $(\alpha=0)$ for $T>T_{C}$. However, for $T<T_{C} \alpha=+1 / 3$ is observed instead of $\alpha=+1 / 4$ for $E u S$.

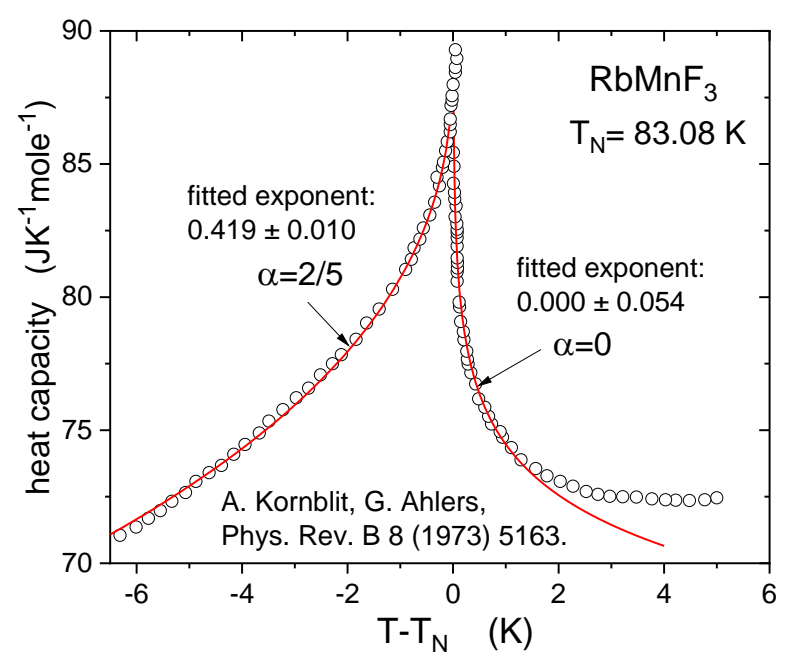

Figure 11. Critical Heat capacity of antiferromagnetic $\mathrm{RbMnF}_{3}$ as a function of the temperature difference to the Néel temperature [21]. Using a three-parameter fit, the heat capacity turns out to be logarithmically divergent for $T>T_{N}$, as for ferromagnetic EuS (Figure 8) and $\mathrm{EuO}$ (Figure 10). For $T<T_{N}$ a particular exponent $(\alpha=+2 / 5)$ is observed.

Figure 12 shows the heat capacity of $\mathrm{NiCl}_{2} \cdot 6 \mathrm{H}_{2} \mathrm{O}$, as a typical example of an antiferromagnet with a low Néel temperature of $5.34 \mathrm{~K}$ only [54]. The asymptotic $\mathrm{T}^{3}$ function at the lowest temperatures includes no absolute constant 
and is characteristic for the heat capacity of the $1 \mathrm{~d}$ Goldstone boson field in magnets with an integer spin [6,7]. Integer spin conforms to the $\mathrm{Ni}^{2+}$ ion with $S=1$. The asymptotic $\mathrm{T}^{3}$ function cannot be confused with the heat capacity of the Debye boson field because its pre-factor is much too large. The Debye boson field dominates the heat capacity in the range $12<\mathrm{T}<20 \mathrm{~K}$ only, where the heat capacity of the magnetic degrees of freedom has strongly decreased. This is outside the temperature window of Figure 12. In contrast to the asymptotic $T^{3}$ function, the $T^{3}$ function of the range $12<\mathrm{T}<20 \mathrm{~K}$ includes an absolute constant of $\sim 2.1 \mathrm{JK}^{-1} \mathrm{~mole}^{-1}$ as it is typical for a nonasymptotic universal power function. The absolute constant can be taken as a measure of the interaction strength between the Debye-bosons and the Goldstone bosons. Note that due to this interaction the heat capacities of the two boson fields do not superimpose but appear alternately as a function of temperature $[3,45]$. The relevant system therefore is clearly defined. By chance the exponent is $\varepsilon=3$ for both systems. The boson field with the larger heat capacity determines the universal exponent. For magnets with a very low ordering temperature such as $\mathrm{NiCl}_{2} \cdot 6 \mathrm{H}_{2} \mathrm{O}$, the asymptotic heat capacity for $\mathrm{T} \rightarrow 0$ is determined by the Goldstone bosons. For magnets with a high ordering temperature, say, $\mathrm{T}_{\mathrm{c}}>100 \mathrm{~K}$, the magnetic heat capacity is weak at low temperatures, and the asymptotic heat capacity for $\mathrm{T} \rightarrow 0$ is determined by the Debye bosons (see Figures 1,4, 6) $[3,45]$. In this case a crossover to the heat capacity of the Goldstone bosons occurs at a sufficiently high temperature at which the dispersion energy of the Debye bosons is higher than the dispersion energy of the Goldstone bosons. Thermal energy then is in the Goldstone boson field. Figure 12 includes the Debye heat capacity of $\mathrm{NiCl}_{2} \cdot 6 \mathrm{H}_{2} \mathrm{O}$, observed in the range $12<\mathrm{T}<20 \mathrm{~K}$ after subtraction of the absolute constant. The Debye temperature calculated from the pre-factor of this $\mathrm{T}^{3}$ function is $\Theta_{D}=331 \mathrm{~K}$.

The interpretation of the fitted critical exponents $\alpha$ in Figure 12 is difficult. For instance, the exponent $\alpha=+1 / 4$ for $\mathrm{T}<\mathrm{T}_{\mathrm{N}}$ of $\mathrm{NiCl}_{2} \cdot 6 \mathrm{H}_{2} \mathrm{O}$ is observed also for $\mathrm{EuS}$ (Figure 8). Note, however, that EuS has a half-integer spin of $\mathrm{S}=7 / 2$ but $\mathrm{NiCl}_{2} \cdot 6 \mathrm{H}_{2} \mathrm{O}$ has an integer spin of $S=1$. On the other hand, $\alpha=-1 / 5$ for $\mathrm{T}>\mathrm{T}_{\mathrm{N}}$ in Figure 12 is a new exponent value, not observed as yet.

The two fitted critical exponents $\alpha$ for $\mathrm{T}>\mathrm{T}_{\mathrm{N}}$ and for $\mathrm{T}<\mathrm{T}_{\mathrm{N}}$ of $\mathrm{CoCl}_{2} \cdot 6 \mathrm{H}_{2} \mathrm{O}$ (Figure 13) agree with those for EuS (Figure 8). This, however, is certainly fortuitous. Unfortunately, in Figure 13 there are no data of the asymptotic power function for $\mathrm{T} \rightarrow 0$ available to evaluate the spin quantum number. It is, however, evident that this power function must have a large exponent. The indicated $\mathrm{T}^{9 / 2}$ function of the heat capacity of the isotropic Goldstone boson field in magnets with an integer spin is the most reasonable assumption and is consistent with the analyses to follow. Crystal field interaction therefore is relevant in $\mathrm{CoCl}_{2} \cdot 6 \mathrm{H}_{2} \mathrm{O}$ and decreases the spin quantum number of the $\mathrm{Co}^{2+}$ ion from $\mathrm{S}=3 / 2$ for the free $\mathrm{Co}^{2+}$ ion to $\mathrm{S}_{\mathrm{eff}}=1$. Agreement of the fitted exponent $\alpha$ with those of EuS with a half-integer spin of $S=7 / 2$ (Figure 8) therefore is amazing.

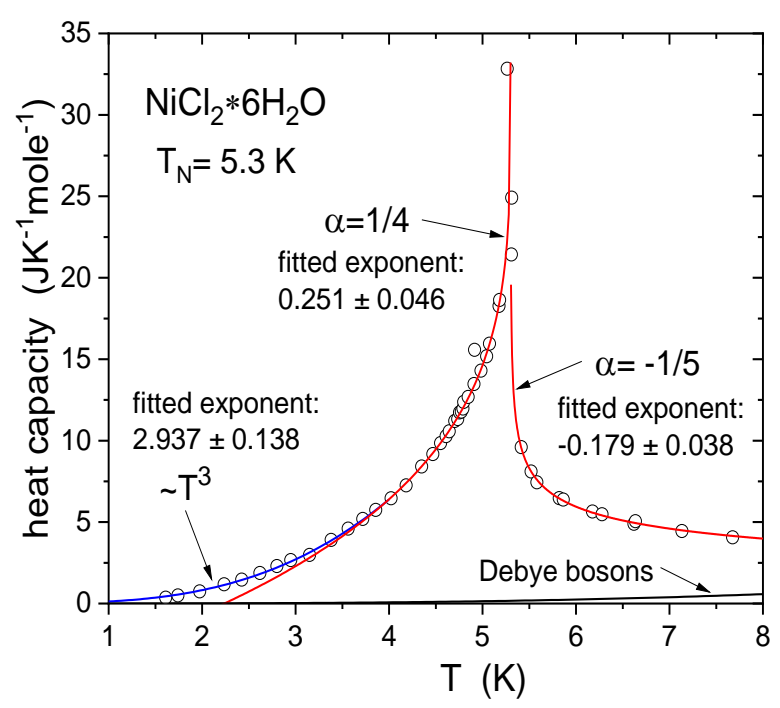

Figure 12. Heat capacity of $\mathrm{NiCl}_{2} \cdot 6 \mathrm{H}_{2} \mathrm{O}$ in the vicinity of the Néel temperature of $T_{N}=5.34 \mathrm{~K}$ as a function of absolute temperature [54]. Three universal power functions can be identified. The low-temperature $T^{3}$ function is due to the $1 d$ Goldstone boson field in magnets with an integer $\operatorname{spin}\left(\mathrm{S}=1\right.$ for the $\mathrm{Ni}^{2+}$ ion). The pre-factor of this $T^{3}$ function is much large than that one of the Debye boson field (curve labeled as Debye bosons).

Comparison of the absolute heat capacity values of $\mathrm{CoCl}_{2} \cdot 6 \mathrm{H}_{2} \mathrm{O}$ (Figure 13) with those of $\mathrm{NiCl}_{2} \cdot 6 \mathrm{H}_{2} \mathrm{O}$ (Figure 12) is very revealing. As can be seen, the heat capacity maximum of $\mathrm{CoCl} 2 \cdot 6 \mathrm{H}_{2} \mathrm{O}$ in Figure 13 is much lower than for $\mathrm{NiCl}_{2} \cdot 6 \mathrm{H}_{2} \mathrm{O}$ in Figure 12, in spite of an assumed identical integer spin of $\mathrm{S}=\mathrm{S}_{\mathrm{eff}}=1$ for both materials. The lower heat capacity of $\mathrm{CoCl}_{2} \cdot 6 \mathrm{H}_{2} \mathrm{O}$ has to be explained by the relevant crystal field interaction in this cobalt compound [45]. The $\mathrm{Co}^{2+}$ ion is known for rather large orbital moment 
contributions in its magnetic moment. The crystal electric field therefore is likely to become relevant, in particular in magnets with a low ordering temperature [45]. Quite generally, the probability for the crystal field to become relevant scales with the ratio of crystal field interaction energy to magnetic interaction energy and therefore is higher for materials with a low ordering temperature [45]. Another well investigated $\mathrm{Co}^{2+}$ compound with a relevant crystal field $\left(\mathrm{S}_{\mathrm{eff}}=1\right)$ is $\mathrm{CoF}_{2}\left(\mathrm{~T}_{\mathrm{N}}=37.85 \mathrm{~K}\right)$ $[45,55,56]$. Note that $\mathrm{CoF}_{2}$ has the lowest ordering temperature compared to the other bi-fluorides with no relevant crystal field: $\mathrm{MnF}_{2}\left(\mathrm{~T}_{\mathrm{N}}=66.5 \mathrm{~K}\right)$, $\mathrm{NiF}_{2}\left(\mathrm{~T}_{\mathrm{N}}=73.2 \mathrm{~K}\right)$ and $\mathrm{FeF}_{2}\left(\mathrm{~T}_{\mathrm{N}}=78.35 \mathrm{~K}\right)$ [56]. The effective spin in the ordered state of $\mathrm{CoF}_{2}$ could be evaluated as $S_{\text {eff }}=1$ using magnetic x-ray diffraction [57], and therefore is lower by $\Delta S=1 / 2$ compared to $\mathrm{S}=3 / 2$ of the free $\mathrm{Co}^{2+}$ ion. In other words, the effect of the relevant crystal field is to excluded one spin state from the dynamics of the ordered state. The number of states therefore remains an integer, and universality is conserved. The observed saturation magnetic moment of 2.21 $\mu_{\mathrm{B}} / \mathrm{Co}$ is perfectly consistent with $\mathrm{S}_{\mathrm{eff}}=1$ $[45,56,57]$.

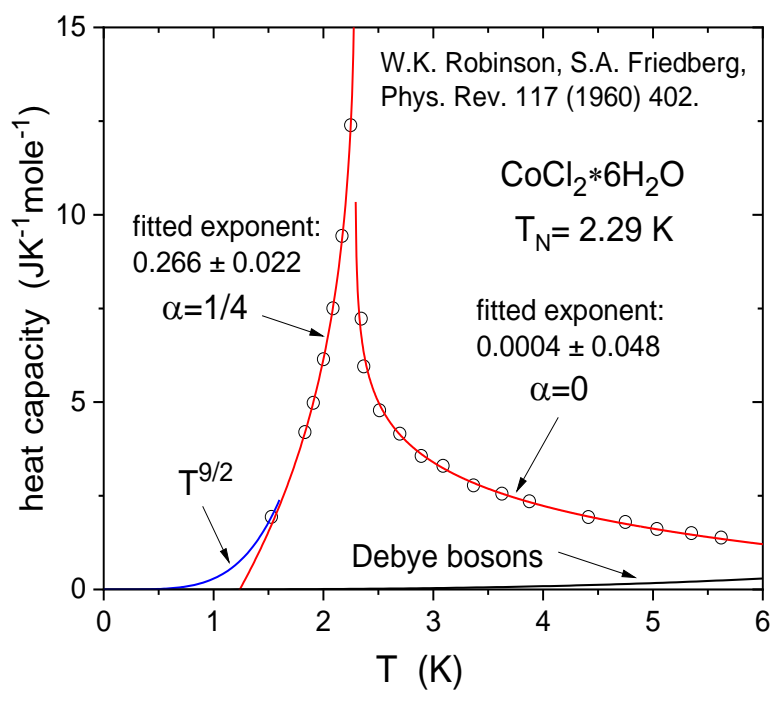

Figure 13. Heat capacity of $\mathrm{CoCl}_{2} \cdot 6 \mathrm{H}_{2} \mathrm{O}$ in the vicinity of the magnetic ordering transition as a function of absolute temperature [54]. By chance, the critical exponents of $\mathrm{CoCl}_{2} \cdot 6 \mathrm{H}_{2} \mathrm{O}$ are identical with those of EuS (Figure 8), in spite of an integer spin of $\mathrm{S}_{\text {eff }}=1$ for $\mathrm{CoCl}_{2} \cdot 6 \mathrm{H}_{2} \mathrm{O}$ but of a half-integer spin of $S=7 / 2$ for $E u S$.

A quantitative analysis of the heat capacity of $\mathrm{CoCl}_{2} \cdot 6 \mathrm{H}_{2} \mathrm{O}$ shows that the magnetic entropy saturates, as for $\mathrm{CoF}_{2}[45,56]$, at a value of $\mathrm{R} \cdot \ln (2)$ instead of $R \cdot \ln (3)$, expected for $S_{\text {eff }}=1$ [54]. The same value of $R \cdot \ln (2)$ is observed also for
$\mathrm{CoCl}_{2} \cdot 2 \mathrm{H}_{2} \mathrm{O}$ [58]. On the other hand, the magnetic entropy of $\mathrm{NiCl}_{2} \cdot 6 \mathrm{H}_{2} \mathrm{O}$ with $\mathrm{S}=1$ and no crystal field interaction, saturates, as expected, at $\mathrm{R} \cdot \ln (3)$ [54]. Note that the spin wave theory treats on nonquantized classical spins and therefore is unable to predict the discrete saturation values for the magnetic entropy at $R \cdot \ln (2 S+1)[45,56]$. Only for paramagnetic salts with discrete magnetic energy levels, the saturation value of the entropy can be calculated as $\mathrm{R} \cdot \ln (2 \mathrm{~S}+1)$.

Saturation of the magnetic entropy at $R \cdot \ln (2)$, irrespective of the value $S_{\text {eff }}$ is typical for the magnetic insulators with a relevant crystal field interaction $[45,54,56]$. This curiosity can be explained by the fact that in magnets with a relevant crystal field interaction, the magnon gap is strongly enhanced. Additionally, for only one spin state eliminated from the dynamics, one further magnon band at a still higher energy occurs [45]. For instance, in $\mathrm{CoF}_{2}$, the measured magnon gap energy corresponds to a temperature of $\sim 54 \mathrm{~K}$ which is larger than the ordering temperature of $\mathrm{T}_{\mathrm{N}}=37.85 \mathrm{~K}$ [55]. The second magnon band, is centered at $\sim 270 \mathrm{~K}$ in $\mathrm{CoF}_{2}$ [55]. A larger gap energy than corresponds to the ordering temperature is known for the conventional Ising magnets only [19]. For instance, in the classical 2d-Ising antiferromagnet $\mathrm{Rb}_{2} \mathrm{CoF}_{4}$, the gap energy is $\sim 300 \mathrm{~K}$ but the ordering temperature is $\sim 100 \mathrm{~K}$ [19]. The large magnon gap in the Ising magnets is atomistic in origin and has to be viewed as a measure of the strong, axial single particle anisotropy that restricts the number of spin states to the two states with $\pm \mathrm{S}$. This view conforms to the classical, non-quantized treatment of the spin.

The magnon gap energy in the here considered magnets with a three-dimensional spin also means anisotropy but is a measure of how strong the spins are coupled to the axis of the one-dimensional boson field within each magnetic domain. As a consequence, for the magnets with a relevant crystal field interaction and therefore with a magnon gap energy of larger than corresponds to the ordering temperature, there are also only the two spin states $\pm \mathrm{S}_{\text {eff }}$ with respect to the axis of the boson field possible. In this way, a saturation value of the entropy of $R \cdot \ln (2)$, irrespective of the value of $S_{\text {eff, can be explained. Only for temperatures }}$ that correspond to the excitation energy of the second magnon band, that is at $\sim 270 \mathrm{~K}$ in $\mathrm{CoF}_{2}$, a crossover to the final entropy value of $\mathrm{R} \cdot \ln (4)$, as it corresponds to the full spin quantum number of $\mathrm{S}=3 / 2$ of the free $\mathrm{Co}^{2+}$ ion, can be expected. This, however, is difficult to evidence because of the 
non-linear superposition of the magnetic and the non-magnetic heat capacity contributions that prevent a quantitative subtraction of the nonmagnetic heat capacity background.

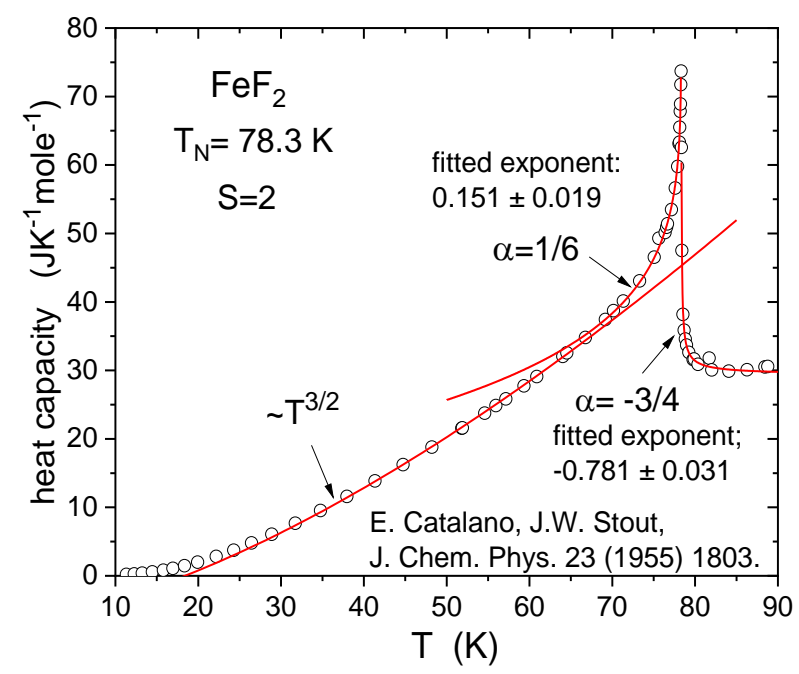

Figure 14. Heat capacity of tetragonal $\mathrm{FeF}_{2}$ for temperatures of lower than the ordering temperature of $T_{N}=78.3 \mathrm{~K}$, as a function of absolute temperature [59].

For the two bi-fluorides $\mathrm{FeF}_{2}$ and $\mathrm{NiF}_{2}$ with tetragonal rutile structure, the spin quantum numbers are both integer $(S=2$ and $S=1$, respectively), and the ordering temperatures are very similar (Figures 14 and 15). Nevertheless, different values for the critical exponents $\alpha$ are observed. Obviously, the absolute value of the spin is important because it determines the proportion between the magnetic and the elastic heat capacity contributions at the critical temperature, and therefore is of importance for the critical exponent $\alpha$. In fact, as a comparison of Figures 14 and 15 shows, the heat capacity peak at $\mathrm{T}_{\mathrm{N}}$ is about twice as large for $\mathrm{FeF}_{2}$ with $\mathrm{S}=2$ compared with $\mathrm{NiF}_{2}$ with $\mathrm{S}=1$. Moreover, in spite of an identical tetragonal lattice symmetry the dynamic symmetry is isotropic in $\mathrm{NiF}_{2}$ but one-dimensional in $\mathrm{FeF}_{2}$ [6]. Thermal decrease of the spontaneous magnetization of $\mathrm{NiF}_{2}$ single crystal material is according to $\mathrm{T}^{9 / 2}$ function $[6,48]$, i.e. $3 \mathrm{~d}$, but according to $\mathrm{T}^{3}$ function for $\mathrm{FeF}_{2}$, i.e. 1d [6]. In other words, for some reasons, the tetragonal lattice anisotropy is not sufficient in $\mathrm{NiF}_{2}$ to induce the crossover to a one-dimensional dynamic behavior. Note that the dynamic symmetry is related to the domain configuration and therefore to the spin orientations. For the tetragonal antiferromagnets $\mathrm{FeF}_{2}$ and $\mathrm{MnF}_{2}$ there is only one domain type along the tetragonal $\mathrm{c}$-axis, and the global boson field therefore is 1d [12,51]. In other words, all ordered spins are along the tetragonal caxis. On the other hand, for the $3 \mathrm{~d}$ material $\mathrm{NiF}_{2}$ the spins were claimed to be oriented in the a-b plane [57]. This, however, would imply that the dynamics of $\mathrm{NiF}_{2}$ is $2 \mathrm{~d}$, and that the spontaneous magnetization should decrease according to a $\mathrm{T}^{2}$ function $[6,7]$. For the observed isotropic dynamic symmetry class of $\mathrm{NiF}_{2}$ single crystal material ( $\mathrm{T}^{9 / 2}$ function) $[6,48]$, a nearly equal distribution of domains, and therefore of spin orientations along all crystallographic axes is compelling. It is evident that the dependence of the dynamic universality class on the domain configuration makes the interpretation of the observed $\alpha$-values additionally complicated. Not for all axial magnets the dynamic symmetry is $1 \mathrm{~d}$.

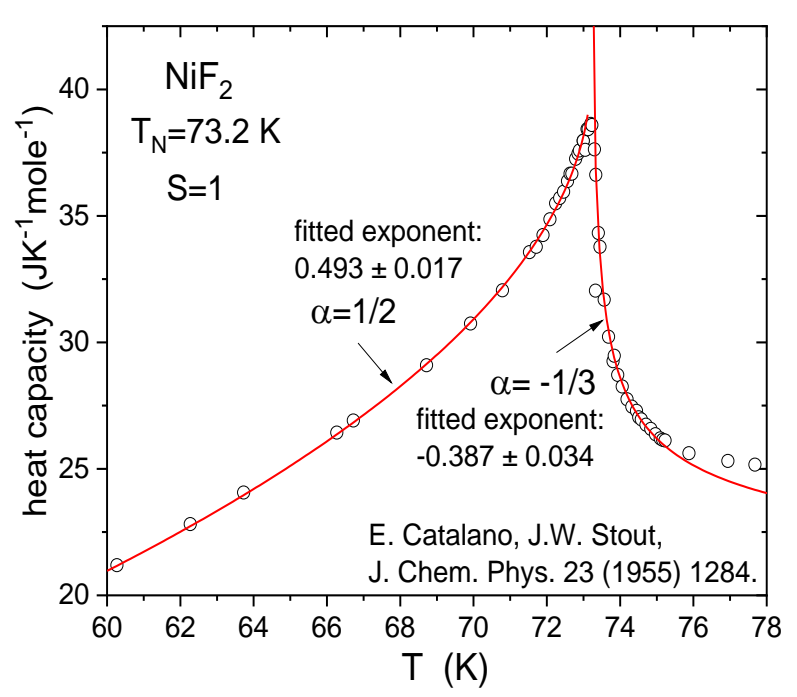

Figure 15. Heat capacity of $\mathrm{NiF}_{2}$ in the vicinity of the magnetic ordering transition at $T_{N}=73.2 \mathrm{~K}$ as a function of absolute temperature [60].

Considering that the critical exponents $\beta, \gamma$ and $v$ are identical for $\mathrm{MnF}_{2}(\mathrm{~S}=5 / 2)$ and for $\mathrm{EuO}$ $(\mathrm{S}=7 / 2)$ [15], and that the ordering temperatures of $\mathrm{T}_{\mathrm{N}}=67.3 \mathrm{~K}\left(\mathrm{MnF}_{2}\right)$ and of $\mathrm{T}_{\mathrm{C}}=69.3 \mathrm{~K}(\mathrm{EuO})$ are very similar, identical values for the critical exponents $\alpha$ can be anticipated. As a comparison of Figure 10 and Figure 16 shows, this is not the case, possibly because of the different lattice structures and the associated different phonon spectra. We should note that the boson field in cubic $\mathrm{EuO}$ is $1 \mathrm{~d}$ in the critical range only but $3 \mathrm{~d}$ below the critical range $[5,7,15]$, while in tetragonal $\mathrm{MnF}_{2}$ the boson field is $1 \mathrm{~d}$ throughout $[6,15]$. In other words, in $\mathrm{EuO}$ (and in EuS) a dimensionality crossover from $1 \mathrm{~d}$ to $3 \mathrm{~d}$ occurs at the crossover from the critical power function with 
the argument $\left|\mathrm{T}_{\mathrm{C}}-\mathrm{T}\right|$ to the $\mathrm{T}^{\varepsilon}$ power function at the critical temperature $\mathrm{T}=0$ [15]. The necessary dynamic coupling of the three domain types at lower temperatures can be explained by an increasing mean-free path of the bosons as a function of a decreasing temperature. The boson fields of adjacent domains then become in denser contact whereby a dynamic coupling between the domains is favored.

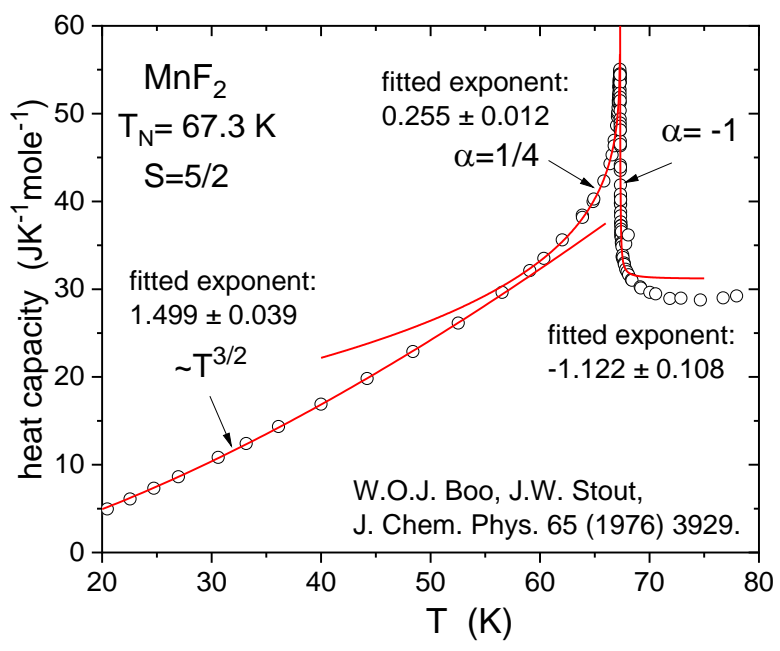

Figure 16. Heat capacity of $\mathrm{MnF}_{2}$ in the vicinity of the magnetic ordering temperature $T_{N}=67.3 \mathrm{~K}$ as a function of absolute temperature [61]. In spite of identical critical exponents $\beta, \gamma$ and $v$ with $E u O$, the critical exponents $\alpha$ for $\mathrm{MnF}_{2}$ and for $\mathrm{EuO}$ are different (compare Figure 10).

On the other hand, as a comparison between Figure 16 and Figure 17 shows the critical exponents $\alpha$ are identical for $\mathrm{MnF}_{2}$ [61] and for $\mathrm{CrF}_{2}$ [62]. This, however, is another puzzle since the spin quantum number is half-integer $(S=5 / 2)$ in $\mathrm{MnF}_{2}$ but integer $(\mathrm{S}=2)$ in $\mathrm{CrF}_{2}$. Integer spin of $\mathrm{S}=2$ for $\mathrm{CrF}_{2}$ is proven by an observed saturation magnetic moment of $3.96 \pm 0.24 / \mathrm{Cr}$ [63]. Consistent with the different spin quantum numbers and with the tetragonal rutile structures [63] is that the thermal decrease of the spontaneous magnetization is according to a one-dimensional boson field, i.e. according to a $T^{5 / 2}$ function for $\mathrm{MnF}_{2}$ with $\mathrm{S}=5 / 2$ but according to a $\mathrm{T}^{3}$ function for $\mathrm{CrF}_{2}$ with $\mathrm{S}=2$ [6].

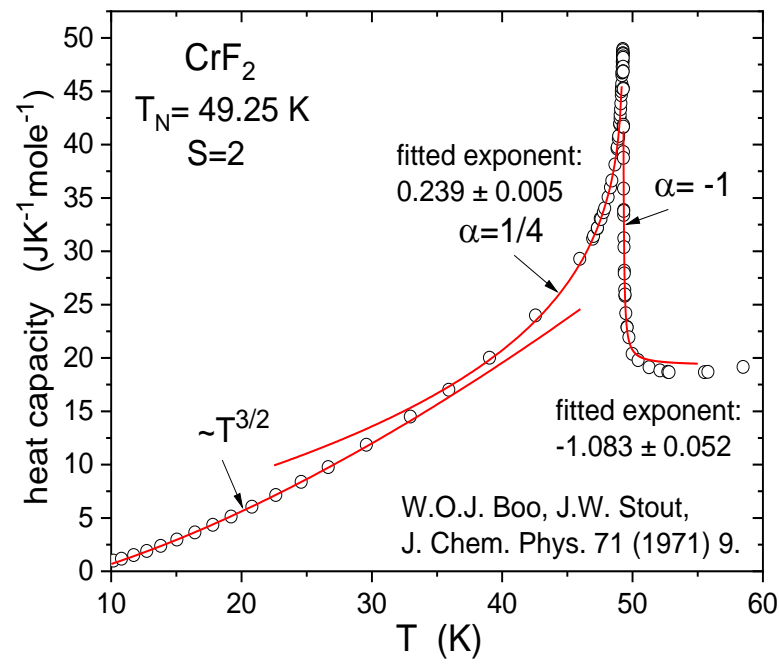

Figure 17. In spite of an integer spin of $S=2$ in $\mathrm{CrF}_{2}$, the same critical exponents are observed as for $\mathrm{MnF}_{2}$ with a half-integer spin of $\mathrm{S}=5 / 2$ (Figure 16) [62]. Consistent with the axial lattice structure, the dynamic symmetry is $1 d$ for both materials [6].

\section{The Heat Capacity for $T>T_{c}$}

The deviations of the high-temperature heat capacity from the Dulong-Petit (D-P) limiting value can be described by the expression $\mathrm{c}=\mathrm{A}+\mathrm{B} \cdot \mathrm{T}^{\varepsilon}$ with $\mathrm{B}$ and $\varepsilon$ negative. As a general observation, the fitted asymptotic heat capacity for $\mathrm{T} \rightarrow \infty$, the fit parameter $\mathrm{A}$, is always larger than the theoretical D-P value that is given by the total number of atomic degrees of freedom $3 \cdot n \cdot N_{L} \cdot k_{B}$ with $n$ as the number of atoms per formula unit, $N_{L}$ as the number of atoms per mole and $\mathrm{k}_{\mathrm{B}}$ as Boltzmann constant.

Figure 18 shows that the high-temperature heat capacity of $\mathrm{MnF}_{2}$ [61] can well be described by $\mathrm{A}=85 \mathrm{JK}^{-1} \mathrm{~mole}^{-1}$ and $\varepsilon=-1$. Note that the theoretical D-P limiting value is $c_{0}=74.85 \mathrm{JK}^{-}$ ${ }^{1} \mathrm{~mole}^{-1}$. Qualitatively, the behavior of the asymptotic heat capacity for $\mathrm{T} \rightarrow \infty$ is similar for the magnetic and for the non-magnetic solids [27]. However, the exponents $\varepsilon$ commonly are different for the two classes of materials. 


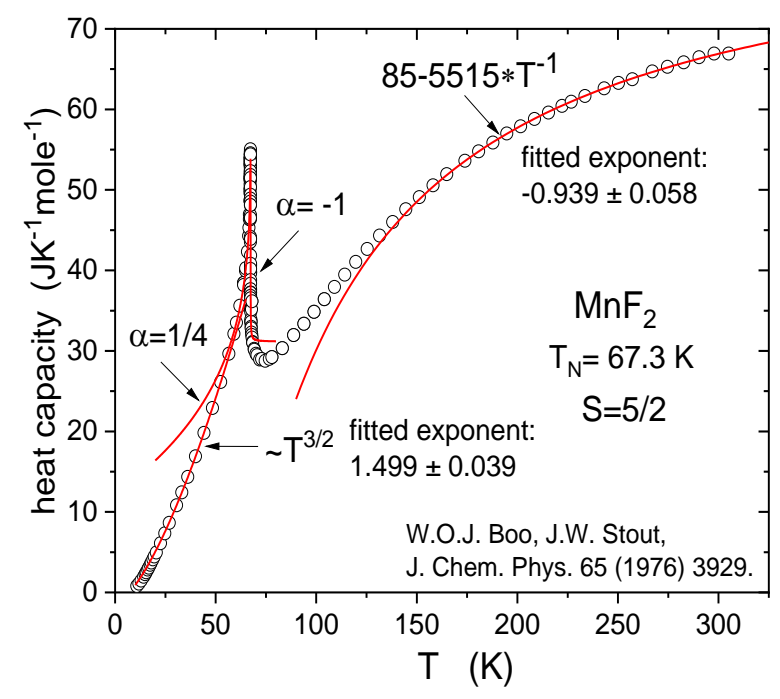

Figure 18. Heat capacity of $\mathrm{MnF}_{2}$ between $10 \mathrm{~K}$ and $300 \mathrm{~K}$ as a function of absolute temperature [61]. The deviations of the high-temperature heat capacity from saturation value of $85 \mathrm{JK}^{-1}$ mole $^{-1}$ can well be described by a $T^{-1}$ function. The critical range at $T_{N}=67.3 \mathrm{~K}$ is better resolved in Figure 16.

A frequently observed exponent is $\varepsilon=-1 / 2$. Figure 19 displays heat capacity data, normalized to the fitted value of the asymptotic parameter A, as a function of $\mathrm{T}^{-1 / 2}$ for a selection of materials with $\varepsilon=-1 / 2$. The lower the absolute value of $\varepsilon$ is, the slower approaches the heat capacity the D-P limit. This is indicative of large magnon energies. It can be seen in Figure 19 that for the temperature range of $\mathrm{T}<300 \mathrm{~K}$, all heat capacity data are considerably below the fitted value for $\mathrm{A}$. The materials of Figure 19, all have an integer (effective) spin: $\mathrm{NiF}_{2}$ has $\mathrm{S}=1[6,48], \mathrm{FeF}_{2}$ has $\mathrm{S}=2$ [6], $\mathrm{CoF}_{2}$ has $\mathrm{S}_{\mathrm{eff}}=1[6,45,56]$ and $\mathrm{MnO}$ has $\mathrm{S}_{\mathrm{eff}}=2$, instead of $\mathrm{S}=5 / 2$ according to a saturation magnetic moment of $4.58 \pm 0.03 \mu_{\mathrm{B}} / \mathrm{Mn}$ [64]. As was shown in [6], magnets with an integer spin have a particular large magnon excitation gap. For $\mathrm{NiF}_{2}$ the magnon gap is $3.86 \mathrm{meV}$, for $\mathrm{FeF}_{2} 6.59$ $\mathrm{meV}$, for $\mathrm{CoF}_{2} 3.54 \mathrm{meV}$ and for $\mathrm{MnO} 3.41 \mathrm{meV}$. The large magnon excitation energies could be responsible for the slow saturation of the heat capacity for $\mathrm{T} \rightarrow \infty$ and for the small absolute value of $\varepsilon$.

The lattice structure is tetragonal for the fluorides but cubic for $\mathrm{MnO}$ (NaCl-type) for $\mathrm{T}>\mathrm{T}_{\mathrm{N}}=144 \mathrm{~K}[28,65]$. The exponent $\varepsilon$ averaged over all four materials is $\varepsilon=-0.479 \pm 0.035$.

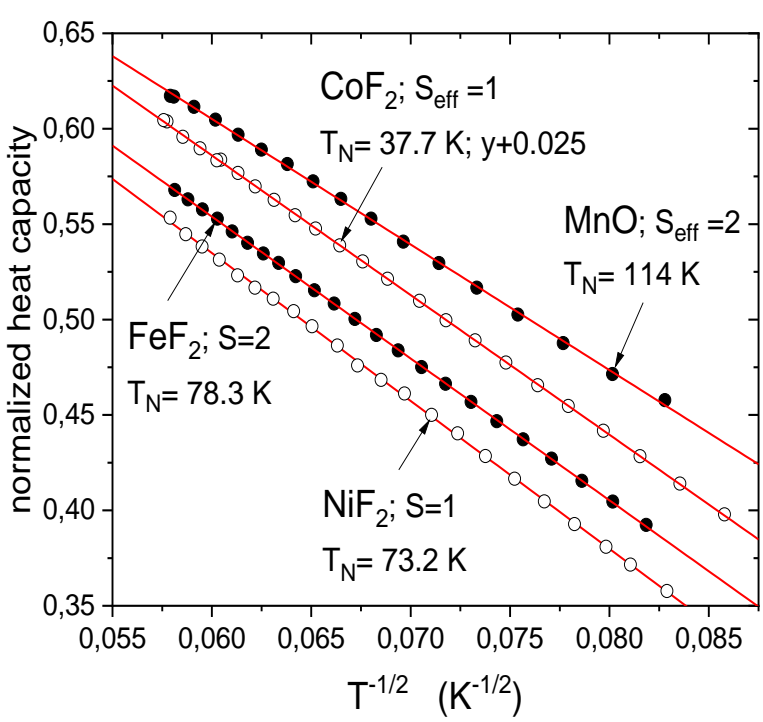

Figure 19. For the selected magnetic solids, the asymptotic heat capacity for $T \rightarrow \infty$ can well be described by the function $c=A+B \cdot T^{\varepsilon}$ with $\varepsilon=-1 / 2$. The ordinate gives the heat capacity normalized to the fitted asymptotic parameter A. All materials have integer (effective) spin (see text).

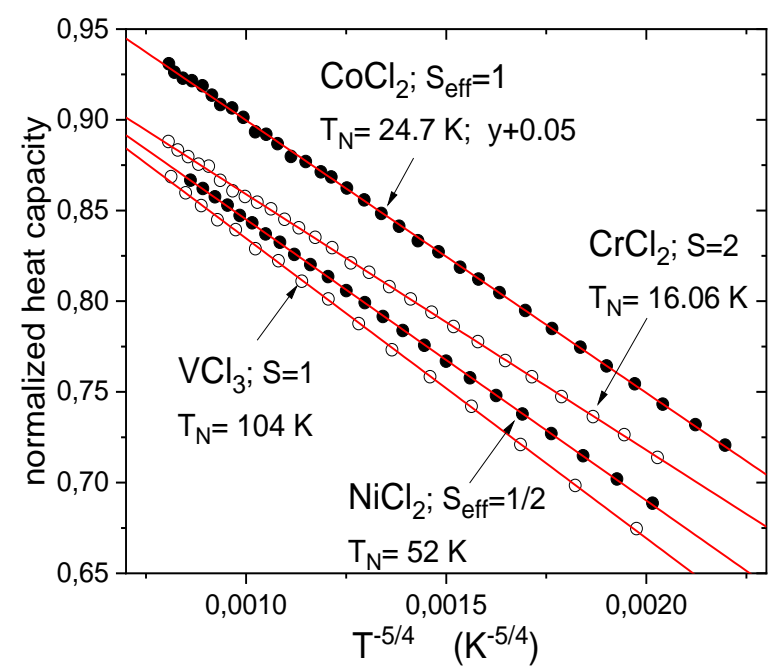

Figure 20. For the selected magnetic solids, the asymptotic heat capacity for $T \rightarrow \infty$ can well be described by the function $c=A+B \cdot T^{\varepsilon}$ with $\varepsilon=-5 / 4$. The ordinate gives the heat capacity normalized to the fitted asymptotic parameter A. For the indicated (effective) spin quantum numbers, see text.

For materials with a larger absolute value of $\varepsilon$, the heat capacity approaches the high-temperature asymptotic value faster. As can be seen in Figure 20 , the heat capacity data for the selected materials with $\varepsilon=-5 / 4$ all are larger than for the materials in Figure 19 with $\varepsilon=-1 / 2$. The effective spin quantum 
numbers given in Figure 20 are, however, not completely clear.

The most likely spin quantum numbers of the materials shown in Figure 20 are $\mathrm{S}_{\mathrm{eff}}=1$ for $\mathrm{CoCl}_{2}$ [66,67], $\mathrm{S}=2$ for $\mathrm{CrCl}_{2}$ [68], $\mathrm{S}=1$ for $\mathrm{VCl}_{3}$ [69] and $\mathrm{S}_{\mathrm{eff}}=1 / 2$ for $\mathrm{NiCl}_{2}$ [6]. The lattice structures are orthorhombic for $\mathrm{CrCl}_{2}$ but rhombohedral for $\mathrm{CoCl}_{2}, \mathrm{VCl}_{3}$ and $\mathrm{NiCl}_{2}$. However, as we have mentioned, for many of the 3d-metal compounds, the spin quantum number of the $3 \mathrm{~d}$-ion is changed with respect to the Hund's rule value by the action of a relevant crystal electric field [45]. Moreover, the effective spin quantum number can depend on sample preparation. This seems to apply to $\mathrm{CoCl}_{2}$. The saturation magnetic moment evaluated for $\mathrm{CoCl}_{2}$ powder material using elastic neutron scattering of $3.1 \pm 0.5 \mu_{\mathrm{B}} / \mathrm{Co}$ is consistent with a regular spin moment of $\mathrm{S}=3 / 2$ of the $\mathrm{Co}^{2+}$ ion [66]. However, analysis of the heat capacity data of the $\mathrm{CoCl}_{2}$ powder sample of Figure 20 gives a saturation value for the magnetic entropy of $R \cdot \ln (2)$ [67]. Since $S=1 / 2$ can be excluded, it follows from $R \cdot \ln (2)$ that the crystal field interaction is relevant (for this $\mathrm{CoCl}_{2}$ sample) and that the spin of the $\mathrm{Co}^{2+}$ ion is $\mathrm{S}_{\text {eff }}=1$ instead of $\mathrm{S}=3 / 2$, as indicated in Figure 20 .

The crystal electric field is relevant also in $\mathrm{NiCl}_{2}$ and reduces the spin of the $\mathrm{Ni}^{2+}$ ion from $\mathrm{S}=1$ to $S_{\text {eff }}=1 / 2$, as can be concluded from observation of a $\mathrm{T}^{3 / 2}$ function in the temperature dependence of the spontaneous magnetization and of the magnon gap energy (AFMR) [6]. The most prominent example of the $\mathrm{T}^{3 / 2}$ universality class of the magnets with a $2 \mathrm{~d}$ boson field and a half-integer spin is $\mathrm{K}_{2} \mathrm{CuF}_{4}$ with $\mathrm{S}=1 / 2$ [6,70]. In summary, the chlorine compounds of Figure 20 seem to have not much in common, except for the exponent $\varepsilon=-5 / 4$. The exponent $\varepsilon$ averaged over all four materials in Figure 20 is $\varepsilon=-1.182 \pm 0.041$.

It is much surprising that the slopes of the different materials in Figure 19 and in Figure 20 are very similar. For the non-magnetic materials discussed in [27], the corresponding slopes scale aproximately with the Debye temperature [27]. Unfortunately, for the magnetic compounds, there is little information on the Debye temperatures. The reason for this is that the correct, the elastic Debye temperature, has to be calculated from the measured sound velocities instead from heat capacity data [71]. This rather laborious task requires accurate low-temperature data for the elastic constants [35]. The elastic Debye temperature can be considerably different from the calorimetric Debye temperature, evaluated from the asymptotic $\mathrm{T}^{3}$ function in the heat capacity [71]. As we have mentioned, only for magnets with a very high ordering temperature the asymptotic heat capacity for $T \rightarrow 0$ is given by the Debye $\mathrm{T}^{3}$ function (Figures 1, 4, 6). On the other hand, the very similar slopes in the Figures 19 and 20 show that in the function $\mathrm{c}=\mathrm{A}+\mathrm{B} \cdot \mathrm{T}^{\varepsilon}$ the exponent $\varepsilon$ and the pre-factor $\mathrm{B}$ are correlated. This supports the view that the universal exponents $\varepsilon$ are determined by the Goldstone bosons and not by the Debye bosons.

As examples of $\varepsilon=0$, i.e. of a logarithmic behavior, Figure 21 and 22 display heat capacity data of $\mathrm{MnO}_{2}$ [72] and of cubic $\mathrm{UO}_{2}$ [39]. The magnetic ordering transitions of the two materials are first order [73]. At first-order transitions, the critical heat capacity seems to be divergent on both sides of $\mathrm{T}_{\mathrm{c}}$. The effective spin of the $\mathrm{Mn}^{4+}$ ion in $\mathrm{MnO}_{2}$ is $\mathrm{S}_{\text {eff }}=1$ instead of $\mathrm{S}=3 / 2$. This can be concluded from observation of a $\mathrm{T}^{3}$ function below the critical range (Figure 21). The lattice structure of $\mathrm{MnO}_{2}$ is tetragonal. In cubic $\mathrm{UO}_{2}$ the spin of the $\mathrm{U}^{4+}$ ion is $\mathrm{S}=1$. This results clearly from observation of $\mathrm{T}^{9 / 2}$ function in the thermal decrease of the spontaneous sublattice magnetization [6]. Interestingly, both materials with a first order magnetic phase transition exhibit the same exponent of $\varepsilon=0$ in the asymptotic power function for $\mathrm{T} \rightarrow \infty$.

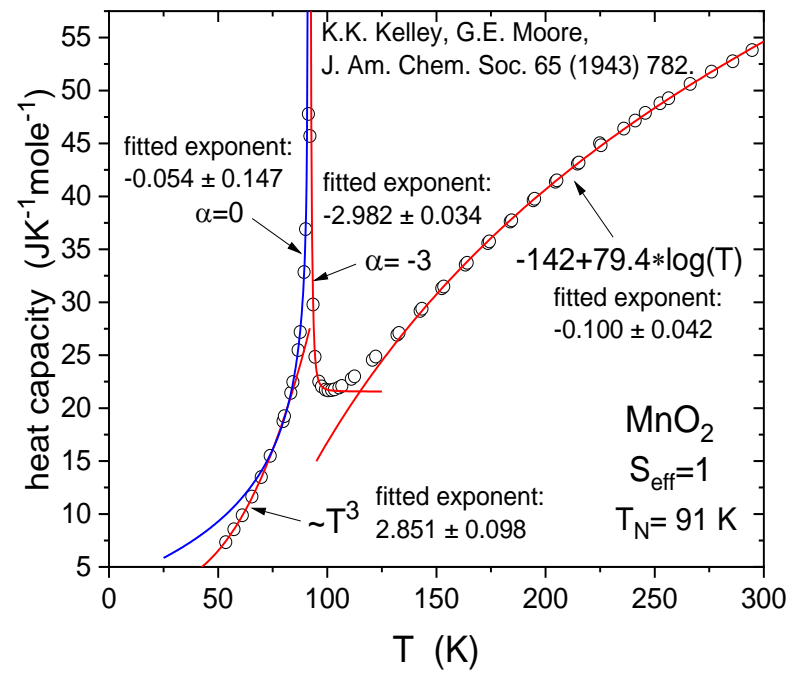

Figure 21. Heat capacity of $\mathrm{MnO}_{2}$ as a function of absolute temperature as an example of $\varepsilon=0$ [72]. The magnetic ordering transition seems to be first order according to a logarithmically divergent critical heat capacity with exponent of $\alpha=0$ on the low-temperature side of $T_{N}$. 


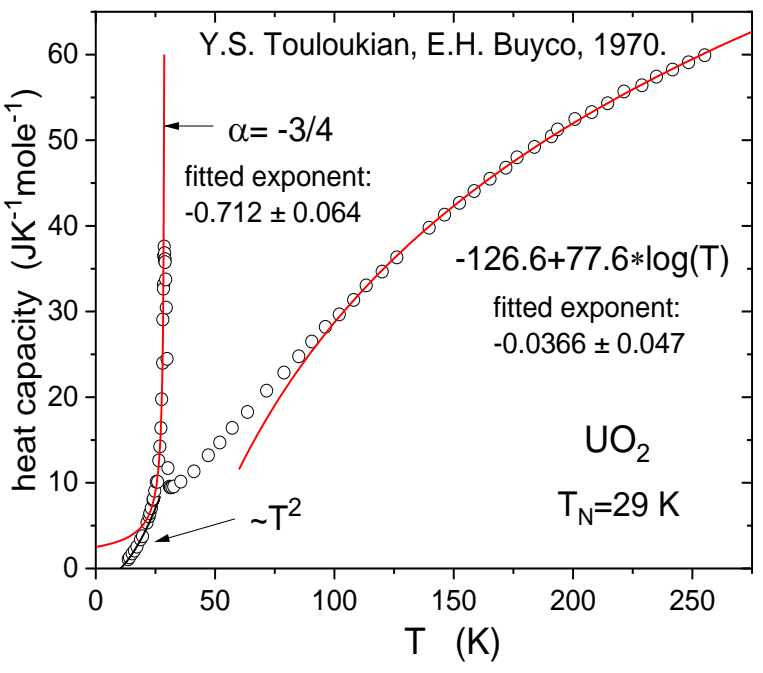

Figure 22. Heat capacity of $\mathrm{UO}_{2}$ as a function of absolute temperature as an example of $\varepsilon=0$ [39]. The magnetic ordering transition of $\mathrm{UO}_{2}$ is strongly first order [73], giving rise to a divergent critical heat capacity with exponent of $\alpha=-3 / 4$ on the low-temperature side of $T_{N}$.

\section{Results}

Within the experimental error limits, the critical exponents $\alpha$ and $\varepsilon$, fitted to the temperature dependence of the heat capacity of the magnetic solids, all seem to be rational numbers but they lack an obvious systematic. One reason for this is that the actual exponent value can depend on the preparation condition of the sample. For instance, the height of the heat capacity peak at $T_{c}$ is wellknown to depend on the crystalline perfection of the sample. The fitted exponents $\alpha$ therefore might also depend on the quality of the sample. Nevertheless, the fitted values for the critical exponent $\alpha$ always are rational numbers within the experimental error limits. In other words, boson dynamics and therefore universality holds also for imperfect samples, and the number of the observed critical exponents remains strongly limited. That universality is independent of the morphology of the sample we know from the amorphous ferromagnets for which no other critical exponents than those known for the single crystals are observed [5,6,32,48]. The universality class, however, can be specific to the individual sample. Moreover, as was shown in [48] the dynamic universality class can be different for single crystal material and for powder samples. This can be understood if the powder grains are single domain particles. The dependence of the universality classes on the absolute size of the sample has been investigated in [32]. Note that in earlier times the heat capacity measurements were performed on large amounts of coarse-grained powder material [12,56,59-62]. In recent studies, small single crystals were sufficient. Another ambiguity is whether the crystal field interaction is relevant, and has changed the spin quantum number and thus the universality class of the spontaneous magnetization. Relevance of the crystal field can also depend on the sample preparation. However, very encouraging is that most of the exponents are reproduced on different materials.

One further complication in the interpretation of the exponents fitted to the heat capacity is that these exponents depend on the unknown coupling strength between the Goldstone bosons and the phonons. Fortunately, the coupling mechanism appears to be quantized $[27,30]$ such that for the heat capacity a non-intrinsic universality arising from the bosons always holds. Due to the different coupling modes, the number of the different observed exponents is larger than the six universality classes of the spontaneous magnetization.

We now list the fitted values for the exponent $\varepsilon$ describing the asymptotic behavior of the heat capacity for $\mathrm{T} \rightarrow \infty$ and for the critical exponents $\alpha$ for $\mathrm{T}>\mathrm{T}_{\mathrm{c}}$ and for $\mathrm{T}<\mathrm{T}_{\mathrm{c}}$. Note our sign convention for $\alpha(\alpha<0$ means a diverging behavior, $\alpha>0$ means a finite heat capacity at $T_{c}$ ). In those cases where the same exponent is observed for different materials the averaged value is added. It can be seen, that within the experimental error limits, the assumption of rational exponent values is confirmed. Some of the exponents are grouped in a rather suggestive way for assumed constant values of the nominator or denominator.

\section{$\varepsilon(\mathbf{T} \rightarrow \infty)$}

$\varepsilon=0\left(\mathrm{UO}_{2}, \mathrm{MnO}_{2}\right)$ average: $\varepsilon=-0.068 \pm 0.045$

$\varepsilon=-2 / 4\left(\mathrm{MnTe}, \mathrm{MnO}, \mathrm{CoF}_{2}, \mathrm{NiF}_{2}, \mathrm{FeF}_{2}\right)$ average:

$\varepsilon=-0.479 \pm 0.035$

$\varepsilon=-3 / 4\left(\mathrm{CuF}_{2}\right.$, Cr-metal $)$ average: $\varepsilon=-0.783 \pm 0.060$

$\varepsilon=-4 / 4\left(\mathrm{MnF}_{2},\right)$

$\varepsilon=-5 / 4\left(\mathrm{CrCl}_{2}, \mathrm{VCl}_{3}, \mathrm{NiCl}_{2}, \mathrm{CoCl}_{2}\right)$ average: $\varepsilon^{=}$

$1.182 \pm 0.041$

$\varepsilon=-6 / 4(\mathrm{EuS})$

$\varepsilon^{=-2 / 3}\left(\mathrm{CrF}_{2}, \mathrm{VF}_{2}, \mathrm{Cr}_{2} \mathrm{O}_{3}\right)$ average: $\varepsilon^{=-}$

$0.669 \pm 0.022$

$\varepsilon=-4 / 3\left(\mathrm{CrCl}_{3}, \mathrm{MnCl}_{2}\right)$ average: $\varepsilon^{=-1.358 \pm 0.086}$

$\varepsilon=-1 / 5\left(\mathrm{NpO}_{2}, \mathrm{EuO}\right)$ average: $\varepsilon=-0.202 \pm 0.038$

$\varepsilon=-2 / 5\left(\mathrm{Fe}_{3} \mathrm{Se}_{4}\right)$

$\varepsilon=-4 / 5(\mathrm{Gd}-\mathrm{metal})$ 


\section{$\alpha\left(\mathbf{T}>\mathbf{T}_{\mathbf{c}}\right)$}

$\alpha=0\left(\mathrm{RbMnF}_{3}, \mathrm{EuO}, \mathrm{EuS}, \mathrm{CrCl}_{2}, \mathrm{Gd}\right)$ average:

$\alpha=0.003 \pm 0.037$

$\alpha=-1 / 4$ (EuTe)

$\alpha=-2 / 4\left(\mathrm{MnS}, \mathrm{MnTe}, \mathrm{FeCl}_{2} \cdot 4 \mathrm{H}_{2} \mathrm{O}\right)$ average: $\alpha=-$

$0.503 \pm 0.070$

$\alpha=-3 / 4\left(\mathrm{CoCl}_{2}, \mathrm{FeF}_{2}, \mathrm{Fe}_{3} \mathrm{Se}_{4}\right)$ average: $\alpha=-$

$0.741 \pm 0.070$

$\alpha=-4 / 4\left(\mathrm{MnF}_{2}, \mathrm{GdCu}_{2}, \mathrm{CoO}, \mathrm{NiO}, \mathrm{TiCl}_{3}, \mathrm{CrF}_{2}\right)$

average: $\alpha=-1.058 \pm 0.071$

$\alpha=-5 / 4\left(\mathrm{CoF}_{2}\right)$

$\alpha=-1 / 3\left(\mathrm{NiF}_{2}\right)$

$\alpha=-1 / 5\left(\mathrm{NiCl}_{2} \cdot 6 \mathrm{H}_{2} \mathrm{O}, \mathrm{Cr}_{2} \mathrm{O}_{3}\right)$ average: $\alpha=-$

$0.188 \pm 0.027$

$\alpha=-2 / 5(\mathrm{GdS})$

\section{$\alpha\left(\mathrm{T}<\mathrm{T}_{\mathrm{c}}\right)$}

$\alpha=1 / 2\left(\mathrm{CoCl}_{2}, \mathrm{MnO}, \mathrm{NiF}_{2}, \mathrm{Cr}_{2} \mathrm{O}_{3}\right)$ average:

$\alpha=0.510 \pm 0.018$

$\alpha=1 / 3\left(\mathrm{EuO}, \mathrm{CoO}, \mathrm{CoF}_{2}\right)$ average: $\alpha=0.344 \pm 0.027$

$\alpha=1 / 4\left(\mathrm{EuS}, \mathrm{MnTe}, \mathrm{MnF}_{2}, \mathrm{CrF}_{2}, \mathrm{NiCl}_{2} \cdot 2 \mathrm{H}_{2} \mathrm{O}\right)$

average: $\alpha=0.254 \pm 0.035$

$\alpha=1 / 5\left(\mathrm{TiCl}_{3}, \mathrm{Gd}\right)$ average: $\alpha=0.209 \pm 0.019$

$\alpha=1 / 6\left(\mathrm{FeF}_{2}\right)$

$\alpha=2 / 3\left(\mathrm{GdCu}_{2}, \mathrm{MnS}, \mathrm{NiCl}_{2}, \mathrm{Fe}_{3} \mathrm{Se}_{4}, \mathrm{CuF}_{2}\right.$,

$\mathrm{Mn}_{2} \mathrm{O}_{3}, \mathrm{CrCl}_{2}$ ) average: $\alpha=0.660 \pm 0.032$

$\alpha=2 / 5\left(\mathrm{GdS}, \mathrm{RbMnF}_{3}\right)$ average: $\alpha=0.411 \pm 0.011$

$\alpha=4 / 5(\mathrm{NiO})$

$\alpha=3 / 4\left(\mathrm{CuCl}_{2}\right)$

\section{Conclusions}

Identification of a clear systematic in the here fitted critical exponents was not possible on the basis of the limited number of the investigated materials. Too many and difficult to estimate parameters influence the exponents of the heat capacity. Continuing studies of more materials appear highly desirable in order to substantiate the rather preliminary conclusions of this work. However, the general importance of boson fields for the dynamics of solids could further be demonstrated, by the here investigated magnetic solids. In a preceding publication this was demonstrated for the non-magnetic solids [27]. Quite generally, in the solids we have to distinguish between the bosons of the elastic, magnetic, electric and metallic degrees of freedom. The excitation spectra of these bosons exist in addition to the well-known atomistic excitations (phonons, magnons, electronic band states...), and cannot be neglected when discussing the dynamics, for instance, the heat capacity. The two types of (quasi)particles and their excitation spectra are distinguished by different translational symmetries (continuous and discrete-periodic). Symmetry conservation implies that the two excitation systems become relevant for the dynamics alternately only. This, however, requires a finite interaction between them in order that thermal energy can change from one to the other system. Only in the metals it is observed that at low-temperatures the heat capacities of the Debye bosons $\left(\sim \mathrm{T}^{3}\right)$ and of the bosons of the metallic continuum $(\sim \mathrm{T})$ superimpose. This is because the two boson types do not interact [26].

Most of the mentioned bosons and their sources are not yet clearly specified. Only the bosons of the continuous elastic solid are well known from practical experience as sound waves. The bosons of the continuous magnetic solid are essentially magnetic dipole radiation generated by the precessing spins [3]. We have every reason to assume that the bosons of the ferroelectric insulators are essentially electric dipole radiation [74]. The bosons of the continuous metallic solids are completely unexplored. All what we know is that the heat capacity of these bosons is a linear function of temperature [25]. Note that the up to know exclusively discussed electronic band states of the metals are the excitations of the discrete translational symmetry of the atomic lattice [75]. By chance, the band theories of the metals also predict a linear-in- $T$ heat capacity for the electronic band states [41,75]. However, the observed low-temperature linear-in $\mathrm{T}$ heat capacity is bosonic in character and has a much larger pre-factor than the $\sim \mathrm{T}$ heat capacity of the band states that occurs at higher temperatures only where the atomistic band states are the relevant excitations.

Universality of the thermodynamics of boson fields means independence of the chemical composition of the solid. For instance, the $\mathrm{T}^{3}$ heat capacity of the Debye boson field is observed for all non-magnetic solids at low temperatures [27]. Only the pre-factor of the $\mathrm{T}^{3}$ function is material specific. In the same way, the same Planck electromagnetic radiation field is emitted by all glowing solids (black bodies) independent of their chemical composition.

For the ballistic propagating bosons there exists no atomic structure. In the elastic case, there is a problem associated with this view. In spite of no atoms, the elastic continuum has a mass density or 
specific gravity. Responsible for the specific gravity of the elastic continuum are, of course, the atoms, that do, however, not occur in the elastic continuum. Sound waves, on the other hand, have no mass but they are possible in a medium with a mass only. The fact that sound waves propagate in a completely identical manner in solids, liquids and gases shows that they are energy degrees of freedom that are completely decoupled from the atoms and their thermal motions.

The atoms and their electronic structures are, however, very important for all boson fields, since they have to be identified as the sources of the bosons. However, spontaneous generation or absorption of bosons by individual atoms are completely unexplored processes. Even for the magnetic case there is no quantitative theory available that would describe the spontaneous generation of magnetic dipole radiation (Goldstone bosons) by the precessing atomic moments. Spontaneous generation or absorption of sound waves by individual atoms is completely non-understood as well. The sources of the Planck electromagnetic radiation field are electric dipoles of unclear provenance.

The atomistic excitations such as phonons or magnons have a finite upper energy limit given by the inter-atomic interaction strengths. For the dispersion energy of the freely propagating bosons there is no general upper energy limit. For instance, the dispersion of the sound waves is a monotonically increasing function of wave-vector for all energies, up to melting point $[27,30]$. As a consequence, for elevated thermal energies viz. temperatures, the dispersion energy of the Debye bosons is larger than for the phonons. It is intuitively clear that under this condition thermal energy is primarily in the atomistic system with the lower dispersion energy. The dynamics therefore is atomistic. This qualitative argument becomes a quantitatively absolute distinction through the symmetry selection principle of relevance [1]. Because of the different translational symmetries of phonons and Debye-bosons, thermal energy can be either in one or the other of the two systems only. This means, the dispersion relation of the sound waves and of the phonons can be populated thermally only alternately. At high-temperatures the phonons are the relevant excitations because they have the lower dispersion energy. However, on approaching the critical temperature $\mathrm{T}=0$ a crossover occurs at which thermal energy changes from the phonon system into the Debye boson field [6]. The heat capacity now exhibits the universal
$\mathrm{T}^{3}$ dependence according to Debye, and the heat capacity of the non-excited phonons is zero. This example visualizes a general principle: upon approaching $\mathrm{T}=0$ or any other finite critical temperature, boson fields become generally the relevant excitations such that the critical dynamics exhibits universality. Probably, all order-disorder phase transitions are driven by boson fields [76,77].

The interactions between the bosons and the atomistic excitations modify the dispersion relations of both systems, and thus affect the critical exponents. This makes any field theory of the dynamics rather complicated. As we have shown recently, the Debye bosons modify the phonon dispersions [27], and the Goldstone bosons modify the magnon dispersions. As a consequence, atomistic theories are generally not able to describe all experimental details on the atomistic length scale correctly. As a consequence of these modifications the heat capacity of the phonons exhibits a non-intrinsic universal temperature dependence, arising from the interactions with the bosons. The here discussed universality in the heat capacity of the magnetic solids has also to be considered as non-intrinsic.

At the magnetic ordering transition, the boson field orders, and the system decomposes into domains, each with a perfect one-dimensional order. A two-dimensional or three-dimensional global boson field results from the coupling of the one-dimensional boson fields of the differently oriented domains and can be read from the number of inequivalent domain orientations. The perfect one-dimensional symmetry within each domain provides strong evidence that the bosons get generated by stimulated emission and therefore are in coherent states. In other words, the generation process of the bosons by stimulated emission appears to be one mechanism for the phenomenon of broken symmetry. Coherence of the bosons is the reason for the long-range and perfectly coherent atomic or spin order. The inter-atomic interactions are not able to stabilize a coherent long-range atomic or spin order.

\section{Acknowledgements:}

I extend my thanks to all of the excellent and ingenious experimentalists that have produced the accurate heat capacity data that turned out to be of fundamental importance for the development of a better understanding of the dynamics of solids. 


\section{References:}

[1] K.G. Wilson, J. Kogut, "The Renormalization Group and the $\varepsilon$ Expansion," Phys. Rep., 12C, 75199, 1974.

[2] J. Goldstone, A. Salam, S. Weinberg, "Broken Symmetries," Phys. Rev., 127, 965-970, 1962.

[3] A. Hoser, U. Köbler, "Boson Fields in Ordered Magnets," Acta Phys. Pol. A, 127, 350-352, 2015.

[4] E. Brézin, J.C. Le Guillou, J. Zinn-Justin, "Discussion of critical phenomena for general nvector models," Phys. Rev. B, 10, 892-900, 1974.

[5] U. Köbler, "Crossover phenomena in the critical range near magnetic ordering transition," J. Magn. Magn. Mater., 453, 17-29, 2018.

[6] U. Köbler, A. Hoser, Experimental Studies of Boson Fields in Solids, Singapore: World Scientific, 2018.

[7] U. Köbler, "Bosonic and magnonic magnon dispersions," J. Magn. Magn. Mater., 502, 166533, 1-18, 2020.

[8] L.J. De Jongh, A.R. Miedema, "Experiments on simple magnetic model systems," Adv. Phys., 23,1-260, 1974.

[9] J.C. Le Guillou, J. Zinn-Justin, "Critical exponents from field theory," Phys. Rev. B, 21, 3976-3998, 1980.

[10] A. Okazaki, K.C. Turberfield, R.W.H. Stevenson, "Neutron Inelastic Scattering Measurements of Antiferromagnetic Excitations in $\mathrm{MnF}_{2}$ at $4.2 \mathrm{~K}$ and at Temperatures up to the Néel Point," Phys. Lett., 8, 9-11,1964.

[11] Y. Shapira, S. Foner, "Magnetic Phase Diagram of $\mathrm{MnF}_{2}$ from Ultrasonic and Differential Magnetization Measurements," Phys. Rev. B, 1 , 3083-3096, 1970.

[12] J.W. Stout, L.M. Matarrese, "Magnetic Anisotropy of the Iron-Group Fluorides," Rev. Mod. Phys., 25, 338-343, 1953.

[13] C.A.M. Mulder, H.L. Stipdonk, P.H. Kes, A.J. van Duyneveldt, L.J. de Jongh, "The Magnetic Phase Diagram of the Quasi TwoDimensional Heisenberg Antiferromagnet $\mathrm{K}_{2} \mathrm{MnF}_{4}$," Physica, 113B, 380-390, 1982.
[14] A. Hoser, U. Köbler, "Functional crossover in the dispersion relations of magnons and phonons," J. Phys.: Conf. Ser., 746, 012062, 1-8, 2016.

[15] U. Köbler, "One-Dimensional Boson Fields in the Critical Range of EuS and EuO," Acta Phys. Pol. A, 128, 398-407, 2015.

[16] U. Köbler, A. Hoser, "Dimensionality in Field Theory and in Spin Wave Theory," Acta. Phys. Pol. A, 127, 356-358, 2015.

[17] L. Onsager, "Crystal Statistics. I. A TwoDimensional Model with an Order-Disorder Transition," Phys. Rev., 65, 117-149, 1944.

[18] H. Ikeda, K. Hirakawa, "Neutron Scattering Study of Two-Dimensional Ising Nature of $\mathrm{K}_{2} \mathrm{CoF}_{4}$," Sol. State Commun., 14, 529-532, 1974.

[19] H. Ikeda, M.T. Hutchings, "Spin wave excitations in a two-dimensional Ising-like antiferromagnet, $\mathrm{Rb}_{2} \mathrm{CoF}_{4}$," J. Phys. C: Solid State Phys., 11, L529-532, 1978.

[20] D.J. Breed, K. Gilijamse, A.R. Miedema, "Magnetic Properties of $\mathrm{K}_{2} \mathrm{CoF}_{4}$ and $\mathrm{Rb}_{2} \mathrm{CoF}_{4}$ : Two-Dimensional Ising Antiferromagnets," Physica, 45, 205-216, 1969.

[21] A. Kornblit, G. Ahlers, "Heat Capacity of $\mathrm{RbMnF}_{3}$ near the Antiferromagnetic Transition Temperature," Phys. Rev. B, 8, 5163-5174 1973.

[22] A. Kornblit, G. Ahlers, "Heat capacity of EuO near the Curie temperature," Phys. Rev. B, 11, 2678-2688, 1975.

[23] E. Scheer, J. Wosnitza, H. v. Löhneysen, "Specific heat of $\mathrm{Eu}_{\mathrm{x}} \mathrm{Sr}_{1-\mathrm{x}}$ Te," Z. Physik B, 85, 7986, 1991.

[24] A. Kornblit, G. Ahlers, E. Buehler, "Heat capacity of EuS near the ferromagnetic transition," Phys. Rev. B, 17, 282-292, 1978.

[25] W.S. Corak, M.P. Garfunkel, C.B. Satterthwaite, A. Wexler, "Atomic Heats of Copper, Silver and Gold from $1^{\circ} \mathrm{K}$ to $5^{\circ} \mathrm{K}$," Phys. Rev., 98, 1699-1708, 1955.

[26] U. Köbler, "On the Thermal Conductivity of Metals and of Insulators," Int. J. Therm., 20, 210218, 2017. 
[27] U. Köbler, "The importance of the Debye bosons (sound waves) for the lattice dynamics of solids," Int. J. Therm., 23, 59-79, 2020.

[28] W.L. Roth, "Magnetic Structures of MnO, FeO, CoO and NiO," Phys. Rev., 110, 1333-1341, 1958.

[29] W. Jauch, M. Reehuis, H.J. Bleif, F. Kubanek, P. Pattison, "Crystallographic symmetry and magnetic structure of CoO," Phys. Rev. B, 64, 052102, 1-3, 2001.

[30] U. Köbler, "On the Distinction between Debye Bosons and Acoustic Phonons," Int. J. Therm., 18, 277-284, 2015.

[31] W. Jauch, M. Reehuis, "Electron density distribution in paramagnetic and antiferromagnetic NiO: A $\gamma$-ray diffraction study," Phys. Rev. B, 70, 195121, 1-8, 2004.

[32] U. Köber, "Sample-size dependent temperature dependence of the spontaneous magnetization," J. Magn. Magn. Mater., 491, 165632, 1-16, 2019.

[33] M. Born, K. Huang, Dynamical Theory of Crystal Lattices, Oxford: Clarendon Press, 1956.

[34] F. Bloch, "Zur Theorie des Ferromagnetismus,“'Z. Physik, 61, 206-219, 1930.

[35] R.F.S. Hearmon, "The elastic constants of crystals and other anisotropic materials," in Landoldt-Börnstein, vol. III/11, pp.1-286, ed. by K.-H. Hellwege and A.M. Hellwege, Berlin: Springer, 1979.

[36] I. Barin, Thermochemical Data of Pure Substances, Weinheim: VCH, 1995.

[37] C. Enss, S. Hunklinger, Low-temperature physics, Berlin: Springer, 2005.

[38] U. Köbler, A. Hoser, "Magnetic Interaction by Exchange of Field Bosons," Acta Phys. Pol. A, 121, 1176-1178, 2012.

[39] Y.S. Touloukian, E.H. Buyco, Thermophysical Properties of Matter, vol. 5, Specific Heat of Nonmetallic Solids, New-York: IFI/Plenum, 1970.

[40] Y.S. Touloukian, E.H. Buyco, Thermophysical Properties of Matter, vol. 4,
Specific Heat of Metallic Elements and Alloys, New-York: IFI/Plenum, 1970.

[41] U. Köbler, V.Yu. Bodryakov, "On the Melting Process of Solids," Int. J. Thermodyn. 18, 200-204, 2015.

[42] S. Klemme, H.S.C. O'Neill, W. Schnelle, E. Gmelin, "The heat capacity of $\mathrm{MgCr}_{2} \mathrm{O}_{4}, \mathrm{FeCr}_{2} \mathrm{O}_{4}$ and $\mathrm{Cr}_{2} \mathrm{O}_{3}$ at low temperatures and derived thermodynamic properties," Amer. Mineralogist, 85, 1686-1693, 2000.

[43] L.M. Corliss, J.M. Hastings, "Magnetic Structure Studies at Brookhaven National Laboratory," J. Physique, 25, 557-562, 1964.

[44] L.M. Corliss, J.M. Hastings, R. Nathans, G. Shirane, "Magnetic Structure of $\mathrm{Cr}_{2} \mathrm{O}_{3}$," J. Appl. Phys., 36, 1099-1100, 1965.

[45] U. Köbler, I. Radelytskyi, H. Szymczak, "Relevant crystal field interaction in the magnetically ordered state," J. Magn. Magn. Mater., 474, 254-268, 2019.

[46] M. Griffel, R.F. Skochdopole, F.H. Spedding, "The Heat Capacity of Gadolinium from 15 to 355 ${ }^{\circ} \mathrm{K}, "$ Phys. Rev., 93, 657-661, 1954.

[47] H.E. Nigh, S. Legvold, F.H. Spedding, "Magnetization and Electrical Resistivity of Gadolinium Single Crystals," Phys. Rev., 132, 1092-1097, 1963.

[48] U. Köbler, "Magnetism of powder samples and of single Crystals," J. Magn. Magn. Mater., 349, 88-94, 2014.

[49] H. Kondoh, "Antiferromagnetic Resonance in NiO in Far-infrared Region," J. Phys. Soc. Jpn., 15, 1970-1975, 1960.

[50] A. Tucciarone, H.Y. Lau, L.M. Corliss, A. Delapalme, J.M. Hastings, "Quantitative Analysis of Inelastic Scattering in Two-Crystal and ThreeCrystal Neutron Spectroscopy; Critical Scattering from RbMnF 3 ," Phys. Rev. B, 4, 3206-3245, 1971.

[51] M.P. Schulhof, R. Nathans, P. Heller, A. Linz, "Inelastic Neutron Scattering from $\mathrm{MnF}_{2}$ in the Critical Region," Phys. Rev. B, 4, 2254-2276, 1971.

[52] B.J.C. van der Hoeven, Jr., D.T. Teaney, V.L. Moruzzi, "Magnetic Equation of State and 
Specific Heat of EuS near the Curie Point," Phys. Rev. Lett., 20, 719-721, 1968.

[53] U. Köbler, Ch. Sauer in Landolt-Börnstein, vol. III/12c, ed. by K.-H. Hellwege, Berlin: Springer, p. 159-371, 1982.

[54] W.K. Robinson, S.A. Friedberg, "Specific Heats of $\mathrm{NiCl}_{2} \cdot 6 \mathrm{H}_{2} \mathrm{O}$ and $\mathrm{CoCl}_{2} \cdot 6 \mathrm{H}_{2} \mathrm{O}$ between $1.4^{\circ}$ and $20^{\circ} \mathrm{K}, "$ Phys. Rev., 117, 402-408, 1960.

[55] R.A. Cowley, W.J.L. Buyers, P. Martel, R.W.H. Stevenson, "Magnetic excitations and magnetic critical scattering in cobalt fluoride," $J$. Phys. C: Solid State Phys., 6, 2997-3019, 1973.

[56] J.W. Stout, E. Catalano, "Heat Capacity of Zinc Fluoride from 11 to $300^{\circ} \mathrm{K}$. Thermodynamic Functions of Zinc Fluoride. Entropy and Heat Capacity Associated with the Antiferromagnetic Ordering of Manganous Fluoride, Ferrous Fluoride, Cobaltous Fluoride and Nickelous Fluoride," J. Chem. Phys., 23, 2013-2022, 1955.

[57] J. Strempfer, U. Rütt, S.P. Bayrakci, Th. Brückel, W. Jauch, "Magnetic properties of transition metal fluorides $\mathrm{MF}_{2}(\mathrm{M}=\mathrm{Mn}, \mathrm{Fe}, \mathrm{Co}$, Ni) via high-energy photon diffraction," Phys. Rev. B, 69, 014417, 1-9, 2004.

[58] T. Shinoda, H. Chihara, S. Seki, "Heat Capacity of $\mathrm{CoCl}_{2} \cdot 2 \mathrm{H}_{2} \mathrm{O}$ between 7 and $120{ }^{\circ} \mathrm{K}$ and its Anomaly Associated with Magnetic Transition," J. Phys. Soc. Jpn., 19, 1637-1648, 1964.

[59] E. Catalano, J.W. Stout, "Heat Capacity and Entropy of $\mathrm{FeF}_{2}$ and $\mathrm{CoF}_{2}$ from 11 to $300{ }^{\circ} \mathrm{K}$. Thermal Anomalies Associated with Antiferromagnetic Ordering," J. Chem. Phys., 23, 1803-1808, 1955.

[60] E. Catalano, J.W. Stout, "Heat Capacity of $\mathrm{NiF}_{2}$ from 12 to $300{ }^{\circ} \mathrm{K}$. Thermodynamic Functions of $\mathrm{NiF}_{2}$. The Thermal Anomaly Associated with the Antiferromagnetic Ordering," J. Chem. Phys., 23, 1284-1289, 1955.

[61] W.O.J. Boo, J.W. Stout, "Heat capacity and entropy of $\mathrm{MnF}_{2}$ from 10 to $300{ }^{\circ} \mathrm{K}$. Evaluation of the contributions associated with magnetic ordering," J. Chem. Phys., 65, 3929-3934, 1976.

[62] W.O.J. Boo, J.W. Stout, "Heat capacity and entropy of $\mathrm{CuF}_{2}$ and $\mathrm{CrF}_{2}$ from 10 to $300{ }^{\circ} \mathrm{K}$. Anomalies associated with magnetic ordering and evaluation of magnetic contributions to the heat capacity," J. Chem. Phys., 71, 9-16, 1979.

[63] J.W. Cable, M.K. Wilkinson, E.O. Wollan, "Neutron Diffraction Studies of Antiferromagnetism in $\mathrm{CrF}_{2}$ and $\mathrm{CrCl}_{2}$," Phys. Rev., 118, 950-955, 1960.

[64] A.K. Cheetham, D.A.O. Hope, "Magnetic ordering and exchange effects in the antiferromagnetic solid solutions $\mathrm{Mn}_{\mathrm{x}} \mathrm{Ni}_{1-\mathrm{x}} \mathrm{O}$," Phys. Rev., B 27, 6964-6967, 1983.

[65] B. Morosin, "Exchange Striction Effects in MnO and MnS," Phys. Rev. B, 1, 236-243, 1970.

[66] M.K. Wilkinson, J.W. Cable, E.O. Wollan, W.C. Koehler, "Neutron Diffraction Investigations of the Magnetic Ordering in $\mathrm{FeBr}_{2}$, $\mathrm{CoBr}_{2}, \mathrm{FeCl}_{2}$ and $\mathrm{CoCl}_{2}, "$ Phys. Rev., 113, 497507, 1959.

[67] R.C. Chisholm, J.W. Stout, "Heat Capacity and Entropy of $\mathrm{CoCl}_{2}$ and $\mathrm{MnCl}_{2}$ from $11^{\circ}$ to $300^{\circ} \mathrm{K}$. Thermal Anomaly Associated with Antiferromagnetic Ordering in $\mathrm{CoCl}_{2}$," J. Chem. Phys., 36, 972-979, 1962.

[68] J.W. Stout, R.C. Chisholm, "Heat Capacity and Entropy of $\mathrm{CuCl}_{2}$ and $\mathrm{CrCl}_{2}$ from $11^{\circ}$ to $300^{\circ} \mathrm{K}$. Magnetic Ordering in Linear Chain Crystals," J. Chem. Phys., 36, 979-991, 1962.

[69] C.H. Shomate, "Heat Capacities at Low Temperatures of $\mathrm{VCl}_{2}$ and $\mathrm{VCl}_{3}$," J. Am. Chem. Soc., 69, 220-221, 1947.

[70] H. Kubo, K. Shimohigashi, I. Yamada, "Magnetization of Two-Dimensional Ferromagnet $\mathrm{K}_{2} \mathrm{CuF}_{4}$," J. Phys. Soc. Jpn., 34, 1687-1687, 1973.

[71] G.A. Alers, "Use of Sound Velocity Measurements in Determining the Debye Temperature of Solids," in Physical Acoustics, ed. by W.P. Mason, vol III B, New-York: Academic Press, pp. 1-42, 1965.

[72] K.K. Kelley, G.E. Moore, "Specific Heats at Low Temperatures of Manganese Carbide and Manganese Dioxide," J. Am. Chem. Soc., 65, 782$785,1943$.

[73] B.C. Frazer, G. Shirane, D.E. Cox, C.E. Olsen, "Neutron-Diffraction Study of Antiferromagnetism in $\mathrm{UO}_{2}, "$ Phys. Rev., 140, A1448-A1452, 1965. 
[74] E. Fatuzzo, W.J. Merz, Ferroelectricity, [76] R.K. Pathria, Statistical Mechanics, $2^{\text {nd }}$ Ed. Amsterdam: North-Holland, 1967. Oxford: Butterworth-Heinemann, 1996.

[75] J. Singleton: Band Theory and Electronic [77] P. Heller, "Experimental investigations of Properties of Solids, Oxford: Univ. Press, 2014. critical phenomena," Rep. Prog. Phys., 30, 731-826, 1967. 